\title{
LOCAL AND NONLOCAL MAGNETIC DIFFUSION AND ALPHA-EFFECT TENSORS IN SHEAR FLOW TURBULENCE
}

\author{
AXEL BRANDENBURG ${ }^{\mathrm{a}, *}$ and DMITRY SOKOLOFF SF $^{\mathrm{a}, \mathrm{b}}$ \\ ${ }^{a}$ Department of Mathematics, University of Newcastle upon Tyne, NE1 7RU, UK; \\ ${ }^{\mathrm{b}}$ Department of Physics, Moscow State University, 119899 Moscow, Russia
}

(Received 28 July 1998; In final form 4 April 2002)

\begin{abstract}
Various approaches to estimate turbulent transport coefficients from numerical simulations of hydromagnetic turbulence are discussed. A quantitative comparison between the averaged magnetic field obtained from a specific three-dimensional simulation of a rotating turbulent shear flow in a slab and a simple one-dimensional alpha-omega dynamo model is given. A direct determination of transport coefficients is attempted by calculating the correlation matrix of different components of the field and its derivatives. This matrix relates the electromotive force to physically relevant parameters like the tensor components of the $\alpha$-effect and the turbulent diffusivity. The $\alpha$-effect operating on the toroidal field is found to be negative and of similar magnitude as the value obtained in previous work by correlating the electromotive force with the mean magnetic field. The turbulent diffusion of the toroidal field is comparable to the kinematic viscosity that was determined earlier by comparing the stress with the shear. However, the turbulent diffusion of the radial field component is smaller and can even be formally negative. The method is then modified to obtain the spectral dependence of the turbulent transport coefficients on the wavenumber. There is evidence for nonlocal behaviour in that most of the response comes from the smallest wavenumbers corresponding to the largest scale possible in the simulation. Again, the turbulent diffusion coefficient for the radial field component is small, or even negative, which is considered unphysical. However, when the diffusion tensor is assumed to be diagonal the radial component of the diffusion tensor is positive, supporting thus the relevance of a nonlocal approach. Finally, model calculations are presented using nonlocal prescriptions of the $\alpha$-effect and the turbulent diffusion. We emphasize that in all cases the electromotive force exhibits a strong stochastic component which make the $\alpha$-effect and the turbulent diffusion intrinsically noisy.
\end{abstract}

Keywords: $\alpha$-effect; Magnetic diffusion; Shear flow turbulence

\section{INTRODUCTION}

In recent years, significant progress has been made in understanding the generation of large scale magnetic fields by dynamo action. One of the outstanding questions is whether such large scale dynamos work in the limit of high conductivity and in the presence of finite amplitude magnetic fields. These questions have so far mostly been addressed in the framework of fully periodic boxes with external forcing

\footnotetext{
*Corresponding author. Present address: NORDITA, Blegdamsvej 17, DK-2100 Copenhagen Ø, Denmark. E-mail: brandenb@nordita.dk
} 
(Cattaneo and Vainshtein, 1991; Vainshtein and Cattaneo, 1992; Cattaneo and Hughes, 1996; Brandenburg, 2001, hereafter referred to as B01). Subsequently, concerns have come up, because periodic boxes have the peculiar property that magnetic helicity is conserved. In that case the $\alpha$-effect may well be 'catastrophically' quenched by finite amplitude magnetic fields if the magnetic Reynolds number is large (Blackman and Field, 2000; B01).

Unfortunately, externally forced simulations with non-periodic, open boundaries do not seem to alleviate the catastrophic quenching problem (Brandenburg and Dobler, 2001, hereafter referred to as BD). This is the reason why there is now an urgent need to consider simulations of naturally driven turbulence. Apart from convection, for which the $\alpha$-effect has been studied extensively (Brandenburg et al., 1990; Ossendrijver et al., 2001), shear flow turbulence that is driven by the magnetorotational instability is another important example (Brandenburg et al., 1995, hereafter referred to as BNST95; Hawley et al., 1996). In the case of naturally driven turbulence, $\alpha$-effect and turbulent diffusivity are no longer scalars but tensors, nor are they just coefficients but integral kernels. The purpose of this paper is to explore these aspects of inhomogeneous turbulence using three-dimensional simulations.

Simulations relevant to astrophysical bodies or to the Earth that include the effects of rotation and large scale velocity shear have recently displayed strikingly coherent spatio-temporal order (Glatzmaier and Roberts, 1995, BNST95). As an example we reproduce in Fig. 1 a space-time diagram (or butterfly diagram in solar physics) of
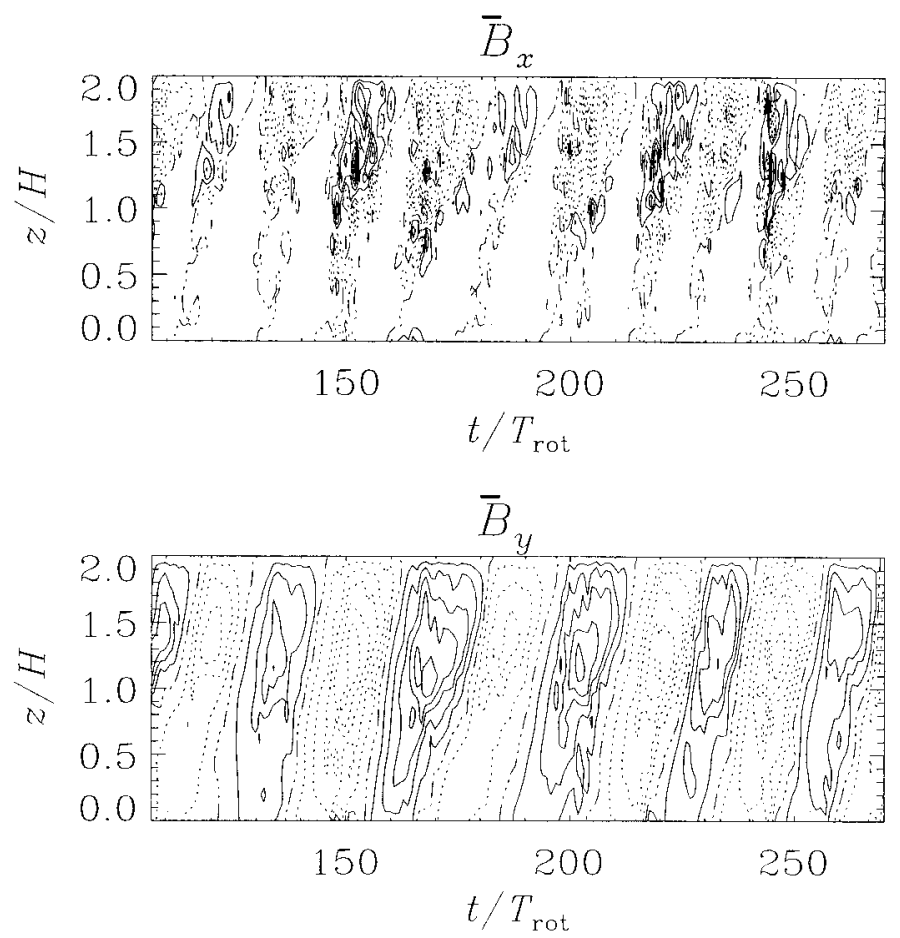

FIGURE 1 Horizontally averaged radial and toroidal magnetic fields, $\overline{B_{x}}$ and $\overline{B_{y}}$ respectively, as a function of time and height, as obtained from the fully three-dimensional simulation of BNST96. Time is given in units of rotational periods, $T_{\text {rot }}$. (No smoothing in $z$ or $t$ is applied.) Dotted contours denote negative values. 
the mean magnetic field of an accretion disc simulation of Brandenburg et al. (1996) (hereafter referred to as BNST96), which is an extended run of BNST95. In this particular simulation (Run 0 of BNST96) the symmetry of the magnetic field has been restricted to even parity, so the computation has been carried out in the upper disc plane, $0<z<L_{z}$, where $L_{z}=2 H$ is the vertical extent of the box, $H$ is the gaussian scale height of the hydrostatic equilibrium density, and $z=0$ corresponds to the equatorial plane.

It is interesting to note that the spatio-temporal behavior obtained from the three-dimensional simulations resembles in many ways what has been obtained earlier using mean-field models. In particular, the magnetic fields of the simulations of Glatzmaier and Roberts (1995) show magnetic field patterns with basic dipole symmetry and occasional field reversal, similar to what is seen in mean-field dynamo models (e.g., Hollerbach et al., 1992; Jones and Wallace, 1992). Of course, the simulations of Glatzmaier and Roberts (1995) are much more realistic and show additional features such as the magnetic dipole inclination. However, we are not aware of a detailed attempt to relate the three-dimensional simulations to mean-field theory. At present there are no estimates of the relevant $\alpha$-effect, or even its sign, and whether it is actually the $\alpha$-effect that is responsible for the large scale field generation in these simulations. Other proposals for causing large-scale field generation include, for example, the incoherent $\alpha$-effect (Vishniac and Brandenburg, 1997), negative magnetic diffusivity effects (Zheligovsky et al., 2001), or effects that conserve magnetic helicity explicitly (Vishniac and Cho, 2001, although there is non-supportive evidence; see Arlt and Brandenburg, 2001).

The aim of this article is to assess the possible relation between simulations and mean-field theory using a local simulation of BNST96 that is readily available to us. This simulation has the advantage of being in cartesian geometry, and one can define averages that are naturally dependent on just one spatial coordinate $(z)$. Furthermore, geometrical or boundary effects are not very pronounced in that model.

In the particular process considered by BNST95 an initial magnetic field of suitable magnitude, but vanishing average $\left(\overline{B_{x}}=\overline{B_{y}}=\overline{B_{z}}=0\right)$, leads after at least thirty rotational periods to a self-sustained turbulent state, which is statistically steady and independent of the detailed initial conditions. This process results from two instabilities, both with positive feedback. There is the magnetic shearing or Balbus-Hawley (1991) instability that generates the turbulence and a dynamo instability that regenerates the magnetic field. The energy that drives the dynamo is constantly being tapped from the large scale velocity shear.

At first glance it may seem inappropriate to invoke a mean-field description in the present case which is highly nonlinear and where the turbulence itself is driven by the dynamo-generated magnetic field. However, we adopt here the view that the mechanism that leads to the generation of large scale magnetic fields is distinct from the feedback cycle that leads to the smaller scale magnetic fields driving the turbulence, which in turn drives the magnetic field. There have been some attempts to model this feedback cycle using a set of phenomenological equations (Tout and Pringle, 1992; Regös, 1997), but these models too involve an $\alpha$-effect that is prescribed in an ad hoc fashion. Unfortunately, these models do not reproduce a number of features seen in the simulations (ratio of poloidal to toroidal fields, magnitude of the accretion torque, role of the Parker instability, and significance of the vertical field.) Also, it should be emphasized that the large scale field seen in BNST95 is really a consequence 
of vertical stratification. Models without stratification (Hawley et al., 1996) do not show large scale fields, although the feedback cycle of dynamo-generated turbulence still works. This confirms that the large scale field generation can indeed be considered as a process operating on top of the otherwise highly nonlinear feedback cycle leading to dynamo-generated turbulence. If this is the case, one might be able to describe the large scale dynamics in terms of averaged equations. This would imply a closure relation between the nonlinear induction term (the electromotive force) and the mean magnetic field. It is this relation which is at the center of the present article.

On the one hand, our findings support basic aspects of the mean-field concept. On the other, when trying to estimate the mean-field transport coefficients ( $\alpha$-effect and turbulent diffusion) it becomes necessary to go beyond simple parameterizations, to adopt tensorial and nonlocal forms of closures, and to allow for stochastic effects. While these results are primarily applicable to local simulations of accretion discs, it will be interesting to make qualitative comparisons with simulations of other systems (geodynamo, solar dynamo).

Our parameterization of transport coefficients depends implicitly on the magnetic field strength, because the data used are from a fully nonlinear calculation. However, we are not able to investigate this dependence in our present approach, because the time-averaged level of magnetic field is rather stable in the model. We should however emphasize that in the present model, where the turbulence is magnetically driven, the magnetic energy is strong (three times in superequipartition with the turbulent energy) and that the turbulent transport coefficients are therefore already strongly affected, and possibly even enhanced, by this strong magnetic field.

Before we begin, we summarize a few important properties of the simulation of BNST96. The calculation was carried out in a cartesian box where overall rotation, radial linear shear and vertical density stratification are included. The boundaries in the toroidal $(y)$ direction are periodic and in the radial $(x)$ direction sliding periodic (Hawley et al., 1995), which is periodic with respect to positions that shift in time. At the top the boundaries are impenetrable and stress free, and the horizontal components of the magnetic field vanish. At the lower boundary of the box a symmetry condition is applied.

We consider the horizontally averaged magnetic field components in the two horizontal directions, $\overline{B_{x}}$ and $\overline{B_{y}}$; see Fig. 1 . The averaged vertical field is conserved, because periodic (or shearing-periodic) boundary conditions are used in the horizontal directions. Since the averaged vertical field vanishes initially, it vanishes at all times, see BNST95. We observe the following remarkable properties of the horizontally averaged field: (i) its sign changes every approximately 15 rotational periods, (ii) at larger heights the reversals occur somewhat later than at lower heights, and (iii) the toroidal and radial fields are out of phase by about $3 \pi / 4$. The latter is more clearly seen in curves showing the averaged field as a function of time (e.g. Fig. 1c in BNST96). Property (ii) can be interpreted as a pattern migration away from the equatorial plane at speed $c>0(c \approx 0.024 \Omega H)$. In addition, there are properties related to the symmetry of the field about the equatorial plane; see Brandenburg (1998) for a discussion of the dependence on boundary conditions (and the striking agreement with predictions from simple $\alpha \Omega$-dynamos).

We begin by discussing the signs and magnitudes of various helicities that are relevant in connection with the $\alpha$-effect. We then compare quantitatively a simple mean field model with the result of the simulations. We also consider the direct 
determination of turbulent transport coefficients allowing for the possibility that the transport coefficients may depend either on $z$ or on the vertical scale (i.e. the vertical wavenumber). Finally we present a model with a scale dependent (or nonlocal) formulation of magnetic diffusion and $\alpha$-effect, as well as a model where strong stochastic fluctuations are included.

\section{MAGNETIC AND KINETIC HELICITIES}

In the light of recent results for $\alpha$ and $\eta_{\mathrm{t}}$ in forced turbulence, it is useful to discuss some relevant properties of the present accretion disc simulations. Firstly, as expected for a rotating stratified medium, the kinetic helicity is negative in the upper disc plane. In B01 the magnitude of the ratio $K_{\text {eff }} \equiv \overline{\boldsymbol{\omega} \cdot \mathbf{u}} / \mid \overline{\left.\mathbf{u}\right|^{2}}$ was found to be equal to the forcing wavenumber, $k_{\mathrm{f}}$. (Here, $\boldsymbol{\omega}=\boldsymbol{\nabla} \times \mathbf{u}$ is vorticity and overbars denote horizontal averaging.) Applying this to the disc simulations we find the effective forcing wavenumber between 2 and 4 in $H \leq z \leq 2 H$, and nearly zero in $0 \leq z \leq H$; see Fig. 1 . These wavenumbers correspond to a length scale of about 3 , which is comparable to the scale of the box. Thus, one cannot expect to see some of the pronounced features of B01 associated with an at least modest amount of scale separation.

In B01 the small scale current helicity, $\overline{\mathbf{j} \cdot \mathbf{b}}$, was of the same sign as $\overline{\boldsymbol{\omega} \cdot \mathbf{u}}$ and of the same magnitude. (Here, $\mathbf{j}=\boldsymbol{\nabla} \times \mathbf{b}$ is the small scale current density in units where the magnetic permeability is unity.) This is not the case in the present simulations. First of all, $\overline{\mathbf{j} \cdot \mathbf{b}}$ is positive near the top of the box in $1.5 \mathrm{H} \leq \mathrm{z} \leq 2 \mathrm{H}$, and at most only about $10 \%$ of $|\overline{\boldsymbol{\omega} \cdot \mathbf{u}}|$. In $0 \leq z \leq 1.5 \mathrm{H}$ the small scale current helicity is essentially fluctuating about zero. However, as in B01, there is also here a tendency for the total current helicity, $\overline{\mathbf{J}} \cdot \overline{\mathbf{B}}+\overline{\mathbf{j}} \cdot \overline{\mathbf{b}}$, to cancel to zero, although not quite, and only near the top boundary where large scale and small scale current helicities tend to have opposite signs. This is however different from the model studied by BD, where open boundary conditions (the same that are used here) were found to yield a modest helicity flux out of the domain, which offsets the otherwise strict steady state balance between large scale and small scale current helicities that was found in B01 and also here. In the present simulations the magnetic helicity flux, $\mathcal{F}$, is however very small; see Fig. 2.

It is somewhat surprising that in the present simulations the resulting magnetic helicity flux is so weak. This could be related to the fact that here, in contrast to $\mathrm{BD}$, the turbulence is driven self-consistently by the resulting magnetic field. Near the boundaries there are relatively sharp gradients in all quantities, which is typical of boundary layer behaviour, and is unlikely to occur in more realistic cases where free turbulent exchange and flows through the boundaries can occur.

The fact that the small scale current helicity is positive indicates that $\alpha$ is negative. The connection between the two is based on a formula given by Keinigs (1983), $\alpha=-\eta \overline{\mathbf{j} \cdot \mathbf{b}} /|\overline{\mathbf{B}}|^{2}$, where $\eta$ is the microscopic magnetic diffusivity ${ }^{1}$. Another more direct estimate for $\alpha$ comes from considering the balance of different induction effects, $\alpha \overline{\mathbf{B}}+\overline{\mathbf{u}} \times \overline{\mathbf{B}}$ on the one side and $\left(\eta+\eta_{\mathrm{t}}\right) \overline{\mathbf{J}}$ on the other. Taking the dot product with $\overline{\mathbf{B}}$

\footnotetext{
${ }^{1}$ We note that the results of B01 are compatible with a version of Keinigs' formula where $\alpha-\eta_{\mathrm{t}} \overline{\mathbf{J}} \cdot \overline{\mathbf{B}} /|\overline{\mathbf{B}}|^{2}$ is equal to $-\eta \overline{\mathbf{j} \cdot \mathbf{b}} /|\mathbf{B}|^{2}$; see Eq. (48) of B01. However, for the dynamo to be excited, the $\eta_{\mathrm{t}}$ term must always be slightly smaller than the $\alpha$ term. Therefore, the sign implied for $\alpha$ is not affected by this generalization.
} 

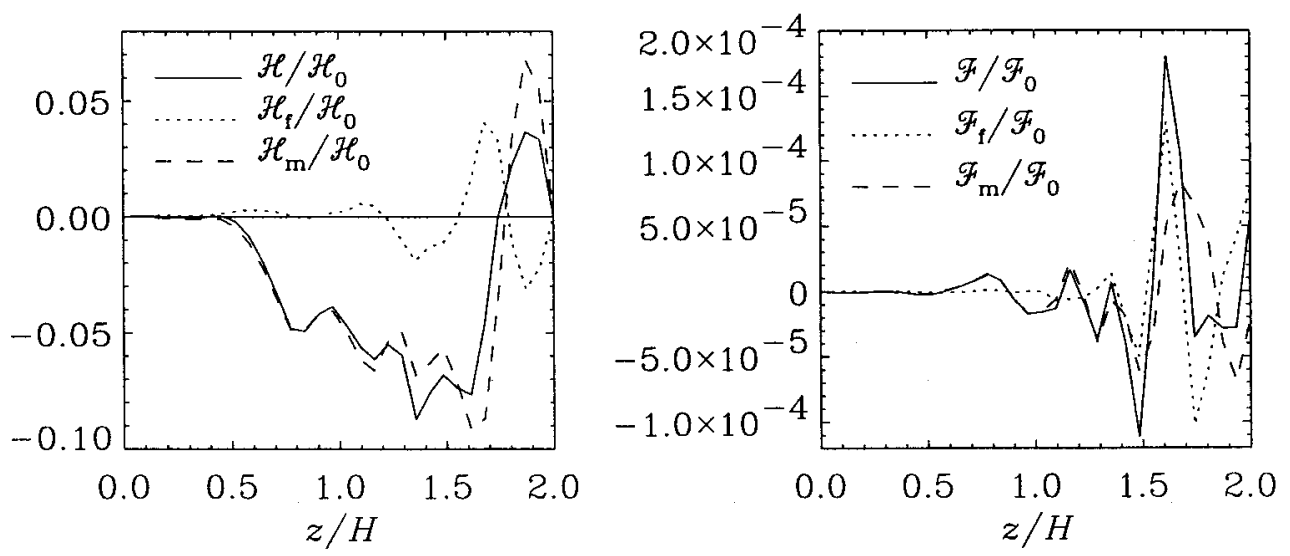

FIGURE 2 Vertical dependence of magnetic helicity $\mathcal{H}=\mathcal{H}_{\mathrm{m}}+\mathcal{H}_{\mathrm{f}}$ and magnetic helicity flux $\mathcal{F}=\mathcal{F}_{\mathrm{m}}+\mathcal{F}_{\mathrm{f}}$, where the subscripts $\mathrm{m}$ and $\mathrm{f}$ denote the contributions from the mean and fluctuating fields. Magnetic helicity is normalized by $\mathcal{H}_{0}=k_{1}^{-1} \int|\mathbf{B}|^{2} \mathrm{~d} V$, where $k_{1}=\pi / L_{z}$ is the smallest wavenumber in the vertical direction. The magnetic helicity flux is normalized by $\mathcal{F}_{0}=\mathcal{H}_{0} \Omega H$, where $H=L_{z} / 2$ is the density scale height of the disc.

shows that $\alpha=+\eta_{\mathrm{t}} \overline{\mathbf{J}} \cdot \overline{\mathbf{B}} /|\overline{\mathbf{B}}|^{2}$. Thus, since $\overline{\mathbf{J}} \cdot \overline{\mathbf{B}}$ is found to be negative we again find that $\alpha$ is negative in the upper disc plane. (The negative sign of $\overline{\mathbf{J}} \cdot \overline{\mathbf{B}}$ is in agreement with a negative sign of the magnetic helicity $\mathcal{H}$ throughout most of the computational domain; see Fig. 2.)

Perhaps the most convincing explanation for the negative $\alpha$ is that intense parts of a flux tube contract (to maintain pressure balance along field lines), but are also most buoyant. If this contraction is stronger than the expansion associated with the rise into a less dense medium, then $\alpha$ will be negative (Brandenburg, 1998). The same result was also obtained by Rüdiger and Pipin (2000) under the assumption that the turbulence is driven primarily by small scale magnetic fields.

Next we compare quantitatively the results of the simulations with a simple mean-field model. Here the negative sign of $\alpha$ follows directly from the fact that the dynamo wave is found to migrate away from the disc plane.

\section{COMPARISON WITH CONVENTIONAL MEAN FIELD DYNAMOS}

In mean-field dynamo theory one uses the averaged induction equation,

$$
\frac{\partial \overline{\mathbf{B}}}{\partial t}=\boldsymbol{\nabla} \times(\overline{\mathbf{u}} \times \overline{\mathbf{B}}+\overline{\delta \mathbf{u} \times \delta \mathbf{B}})
$$

where overbars denote horizontal averages and the deltas denote fluctuations. Our horizontal averages may be associated with ensemble averages in analytical studies. In astrophysical bodies with large scale shear, $\overline{\mathbf{u}}=\overline{\mathbf{u}}(\mathbf{x})$, e.g. differential rotation, a mean component of the magnetic field is generated in the direction of the shear. However, this only works as long as there is a mechanism replenishing the magnetic field component in the cross stream direction. Such a field could arise from the correlation term 
$\overline{\delta \mathbf{u} \times \delta \mathbf{B}}$, the "turbulent" electromotive force, which we denote by $\mathcal{E}$. Early work since the 1960 s and 1970 s has established the following form for $\mathcal{E}$ :

$$
\mathcal{E}_{i}=\alpha_{i j} \overline{B_{j}}+\eta_{i j k} \partial \overline{B_{j}} / \partial x_{k}
$$

(Roberts and Soward, 1975; Krause and Rädler, 1980; see also Steenbeck et al., 1966 for an early reference, and Parker, 1955 for the original formulation of the idea). In this representation one assumes that the averaged magnetic field $\overline{\mathbf{B}}$ is smooth so that higher derivatives in the Taylor expansion (2) do not enter. It is then assumed that the functional relationship between $\mathcal{E}$ and $\overline{\mathbf{B}}$ is local. We return to the applicability of a local relationship in the next section.

An apparently less general formulation of the diffusion term in Eq. (2) is

$$
\mathcal{E}_{i}=\alpha_{i j} \overline{B_{j}}-\eta_{i j}^{*} \overline{J_{j}}
$$

where diffusion works only via the current density $\mathbf{J}=\boldsymbol{\nabla} \times \mathbf{B}$ (in units where the magnetic permeability is unity). However, the two cases are here equivalent, because in Eq. (2) only the index $k=z$ gives a nonvanishing contribution (horizontal derivatives of horizontal averages vanish in our case!). Therefore, the matrices $\eta_{i j z}$ and $\eta_{i j}^{*}$ contain the same information. In particular,

$$
\left(\begin{array}{ll}
\eta_{x x}^{*} & \eta_{x y}^{*} \\
\eta_{y x}^{*} & \eta_{y y}^{*}
\end{array}\right)=\left(\begin{array}{ll}
\eta_{x y z} & -\eta_{x x z} \\
\eta_{y y z} & -\eta_{y x z}
\end{array}\right) .
$$

The advantage of formulation (3) is that it is straightforward to ensure that turbulent diffusion does indeed lead to a decrease of magnetic energy. This is the case when the matrix $\eta_{i j}^{*}$ is positive definite, i.e. $\eta_{i j}^{*} J_{i} J_{j}>0$. A necessary condition for this is $\eta_{x x}^{*}>0$ and $\eta_{y y}^{*}>0$. Of particular interest is the case where $\eta_{i j}^{*}$ is diagonal, i.e. $\eta_{x y}^{*}$ and $\eta_{y x}^{*}$ are assumed to vanish. Below we shall consider both representations, (2) and (3) with $\eta_{x y}^{*}=\eta_{y x}^{*}=0$, and correspondingly we use different notation in the two cases, $\eta_{i j z}$ and $\eta_{i j}^{*}$, respectively. We emphasize that Eq. (4) does not hold if the off-diagonal components of $\eta_{i j}^{*}$ are put to zero, whilst $\eta_{i j z}$ is allowed to have all four components different from zero.

In principle there could be an additional gradient term in the expression for $\mathcal{E}$ that would not affect the evolution of the field, but would modify the fitting procedure for the transport coefficient. (We are grateful to Rogachevskii and Kleeorin (2001) who attracted our attention to this possibility.) In the present case where averages depend only on $z$, such gradient terms correspond simply to a constant vector, $\mathbf{E}_{0}$; see, e.g., BD. However, in the present case the horizontal components of $\mathcal{E}$ are antisymmetric about the midplane and have to vanish there. Since the terms on the right hand side of Eqs. (2) and (3) are also zero on $z=0$ we must have $\mathbf{E}_{0}=\mathbf{0}$.

Now in order that the poloidal field can be replenished it is important to have a nonvanishing component of $\alpha_{y y}$. The magnitude of $\alpha_{y y}$ has to be sufficiently large to overcome the effects of (turbulent) diffusion that arise from $\eta_{\mathrm{t}} \epsilon_{i j k}$, the isotropic part of $\eta_{i j k}$.

In BNST95, and later in Brandenburg and Donner, 1997, the value of $\alpha_{y y}$ was roughly estimated in the following way. It turned out that $\mathcal{E}_{y}$ and $\overline{B_{y}}$ are correlated. The slope of the least-square fit gives a first estimate for $\alpha_{y y}$. It was found that $\alpha_{y y}$ is 
negative in the upper disc plane and positive in the lower, and its magnitude was about $0.001 \Omega H$, where $\Omega=2 \pi / T_{\text {rot }}$ is the local angular velocity and $H$ the gaussian density scale height of the disc. In these articles no explicit estimate for $\eta_{\mathrm{t}}$ was given, but one would expect that $\eta_{\mathrm{t}}$ is comparable to the turbulent kinematic viscosity $v_{\mathrm{t}}$, which was found to be approximately $0.005 \Omega H^{2}$. The negative sign of $\alpha$ in the upper disc plane was confirmed independently by Ziegler and Rüdiger (2000), who used a different code. Their simulations also produced a large scale field. On the other hand, the simulations of Miller and Stone (2000) for a taller box did not show evidence for large scale dynamo action. It is possible that in their case the effective magnetic Reynolds number is larger and therefore the time scale after which a large scale field can be established would be longer. This effect would be even more pronounced if the numerical diffusion operator has hyper-diffusive properties (Brandenburg and Sarson, 2002).

In writing down the mean field equations for the horizontally averaged fields, we reiterate that because of periodic boundary conditions in the horizontal directions the averaged vertical field $\overline{B_{z}}$, remains unchanged, and since $\overline{B_{z}}=0$ initially, it remains so for all times. Thus, we have only two equations for the mean fields in the $x$ and $y$ directions:

$$
\begin{gathered}
\frac{\partial \overline{B_{x}}}{\partial t}=-\frac{\partial \mathcal{E}_{y}}{\partial z}, \\
\frac{\partial \overline{B_{y}}}{\partial t}=\frac{\partial \mathcal{E}_{x}}{\partial z}-q \Omega \overline{B_{x}} .
\end{gathered}
$$

The effect of molecular diffusion is here subsumed into the definition of $\mathcal{E}$. On the boundaries we assume

$$
\frac{\partial \overline{B_{x}}}{\partial z}=\frac{\partial \overline{B_{y}}}{\partial z}=0 \quad \text { on } z=0 ; \quad \overline{B_{x}}=\overline{B_{y}}=0 \quad \text { on } z=L_{z} .
$$

This is equivalent to the conditions used in the three-dimensional simulations for the non-averaged magnetic field. The last term in Eq. (6) arises from the local velocity shear, $\overline{u_{y}}(x)=-q \Omega x$. In the present case of keplerian rotation we have $q=3 / 2$; for estimates of $\alpha_{y y}$ for different values of $q$ see Brandenburg and Donner (1997). The simplest parameterization that leads to dynamo action, balanced by diffusion, would be

$$
\begin{gathered}
\mathcal{E}_{x}=\eta_{\mathrm{t}} \frac{\partial \overline{B_{y}}}{\partial z}, \\
\mathcal{E}_{y}=\alpha_{y y} \overline{B_{y}}-\eta_{\mathrm{t}} \frac{\partial \overline{B_{x}}}{\partial z} .
\end{gathered}
$$

It is instructive to consider first free wave solutions of the form $e^{\lambda t+i k z}$, ignoring thus the boundaries. We now assume $k>0$. The dispersion relation is

$$
\lambda=(1 \pm i)\left|\frac{3}{4} \Omega \alpha_{y y} k\right|^{1 / 2}-\eta_{\mathrm{t}} k^{2}
$$



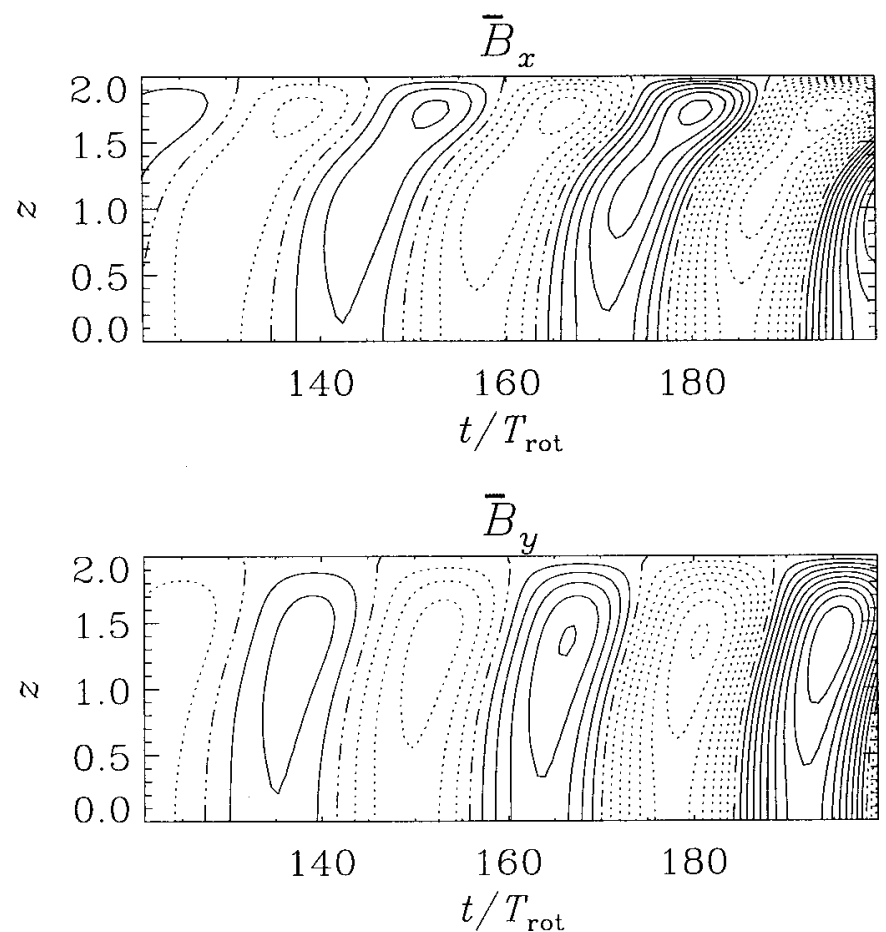

FIGURE 3 Horizontally averaged radial $\left(\overline{B_{x}}\right)$ and toroidal $\left(\overline{B_{y}}\right)$ magnetic fields as a function of time (in rotational periods) and height, $z$, as obtained from the one-dimensional dynamo model. Dotted contours denote negative values.

where the two signs are respectively for positive and negative values of $\alpha_{y y}$. The marginally excited solution $(\operatorname{Re} \lambda=0)$ can be written as

$$
\overline{B_{x}}=A \sin k(z-c t), \quad \overline{B_{y}}=\sqrt{2} A\left|\frac{c}{\alpha_{y y}}\right| \sin \left[k(z-c t) \pm \frac{3}{4} \pi\right],
$$

where $A$ is the amplitude (undetermined in linear theory), and $c$ is the wave speed,

$$
c \equiv-\frac{\operatorname{Im} \lambda}{k}=-\alpha_{y y}\left|\frac{3 \Omega}{4 k \alpha_{y y}}\right|^{1 / 2}=\mp \eta_{\mathrm{t}} k
$$

Note here that $c>0$ (as seen in the simulations, cf. Fig. 1) requires $\alpha_{y y}<0$. This is consistent with the sign of $\alpha_{y y}$ obtained earlier by means of correlating $\mathcal{E}_{y}$ with $\overline{B_{y}}$ (BNST95, Brandenburg and Donner, 1997).

In Fig. 3 we present the result of a numerical integration of (5)-(9), where the boundaries are now taken into account. We chose

$$
\alpha_{y y}=-0.001 \Omega z, \quad \eta_{\mathrm{t}}=0.005 \Omega H^{2},
$$

with $L_{z}=2 H$, and normalize to $\Omega=H=1$, so the rotational period is then $T_{\text {rot }}=2 \pi$. 

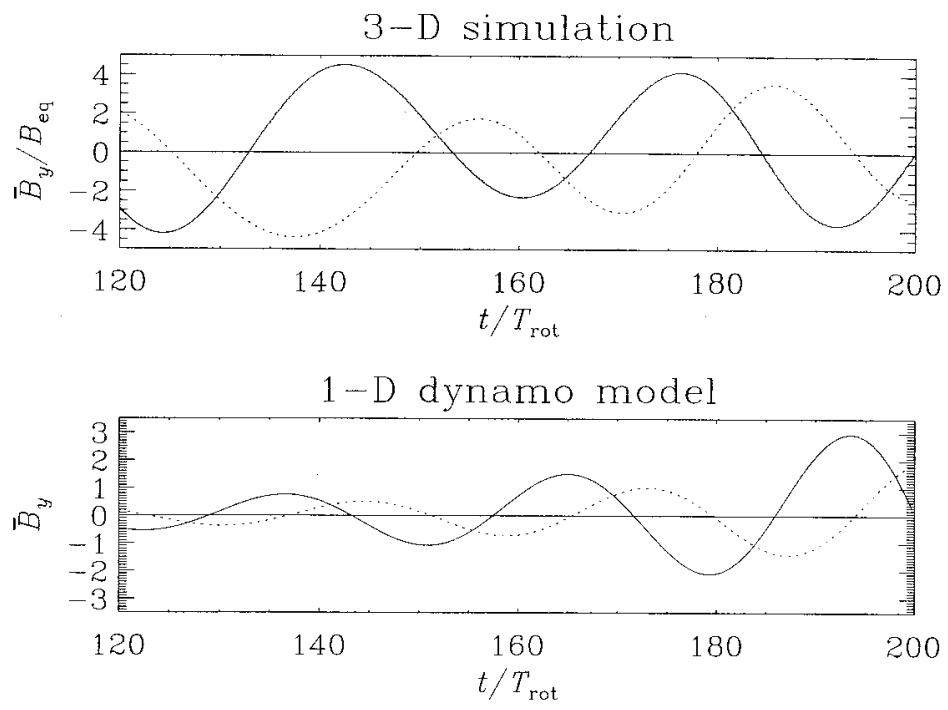

FIGURE 4 Comparison of $\overline{B_{x}}$ (dotted lines) and $\overline{B_{y}}$ (solid lines) as functions of time for $z=1$ for the simulation and the model. Note the similarity in the phase shifts between $\overline{B_{x}}$ and $\overline{B_{y}}$ in the two cases. In both panels $\overline{B_{x}}$ has been scaled up by a factor of 20 . Note that both the model and the simulation have a similar amplitude ratio between the two fields. The horizontally averaged toroidal magnetic field in the simulation is given in units of the equipartition value, $B_{\text {eq }}=\left\langle 4 \pi \rho|\mathbf{u}|^{2}\right\rangle^{1 / 2}$. The model on the other hand is linear and the amplitude is therefore undetermined. The field is growing in time, showing that the mean-field dynamo model is supercritical.

The model reproduces roughly the average behavior of the magnetic field in the three-dimensional simulation. Of course, the simulation shows strong fluctuations, which are not explicitly present in mean-field theory. In the simulations fluctuations are natural because the flow is turbulent and horizontal averages are only an approximation to ensemble averages. Nevertheless the agreement between the mean-field model and the turbulence simulation is striking and even quantitative: the cycle period is $2 \times 15$ rotational periods, the migration speed is positive, although about three times too fast $(c \approx+0.07 \Omega H)$, and the phase difference between $\overline{B_{x}}$ and $\overline{B_{y}}$ is about $3 \pi / 4$ (Fig. 4). Also the amplitude ratio between $\overline{B_{x}}$ and $\overline{B_{y}}$ is similar for the simulation and the model (about 1/30). Thus, the only major disagreement is in the migration speed, which is too fast in the model compared to the simulation.

Our estimates of turbulent transport coefficients in Eq. (13) have been purely phenomenological. What is missing is an analysis of the validity of the expressions (2) or (3). This will be attempted in the following section.

\section{FITTING THE FUNCTIONAL FORM OF $\mathcal{E}$}

In this section we attempt a direct determination of turbulent transport coefficients. We now allow alpha and turbulent diffusivity to be tensors whose components depend either on $z$ (local approach) or on the vertical scale or wavenumber (nonlocal approach). In both approaches we also consider the case where $\eta_{i j}^{*}$ is purely diagonal. 
Here and elsewhere primes on $B_{i}$ and $\mathcal{E}_{i}$ denote $z$-derivatives and dots denote time derivatives. We also drop the overbars on $B_{x}$ and $B_{y}$, which denote now horizontally averaged fields.

\subsection{A Local Formulation}

The underlying turbulence is anisotropic and therefore $\alpha$-effect and turbulent diffusivity are really tensors. From (2) we have

$$
\begin{aligned}
\mathcal{E}_{x} & =\alpha_{x x} B_{x}+\alpha_{x y} B_{y}+\eta_{x x z} B_{x}^{\prime}+\eta_{x y z} B_{y}^{\prime}, \\
\mathcal{E}_{y} & =\alpha_{y x} B_{x}+\alpha_{y y} B_{y}+\eta_{y x z} B_{x}^{\prime}+\eta_{y y z} B_{y}^{\prime} .
\end{aligned}
$$

Since we know $\mathcal{E}$ and $\mathbf{B}$ at different times we can determine the eight coefficients $\alpha_{i j}$ and $\eta_{i j z}$ by forming moments with $B_{x}, B_{y}, B_{x}^{\prime}$ and $B_{y}^{\prime}$. This gives $2 \times 4$ equations for the 8 unknowns, $\alpha_{i j}$ and $\eta_{i j z}$. The reason why the third index on $\eta_{i j k}$ is always $z$ is because only the $z$-derivatives of $B_{i}$ are nonvanishing. This in turn is because the horizontally averaged fields are independent of $x$ and $y$ (see previous section).

The full system of equations can be written in the form of two matrix equations,

$$
\mathbf{E}^{(i)}(z)=\mathbf{M}(z) \mathbf{C}^{(i)}(z), \quad i=x, y,
$$

with the matrix

$$
\mathbf{M}=\left(\begin{array}{cccc}
\left\langle B_{x} B_{x}\right\rangle & \left\langle B_{x} B_{y}\right\rangle & \left\langle B_{x} B_{x}^{\prime}\right\rangle & \left\langle B_{x} B_{y}^{\prime}\right\rangle \\
\left\langle B_{y} B_{x}\right\rangle & \left\langle B_{y} B_{y}\right\rangle & \left\langle B_{y} B_{x}^{\prime}\right\rangle & \left\langle B_{y} B_{y}^{\prime}\right\rangle \\
\left\langle B_{x}^{\prime} B_{x}\right\rangle & \left\langle B_{x}^{\prime} B_{y}\right\rangle & \left\langle B_{x}^{\prime} B_{x}^{\prime}\right\rangle & \left\langle B_{x}^{\prime} B_{y}^{\prime}\right\rangle \\
\left\langle B_{y}^{\prime} B_{x}\right\rangle & \left\langle B_{y}^{\prime} B_{y}\right\rangle & \left\langle B_{y}^{\prime} B_{x}^{\prime}\right\rangle & \left\langle B_{y}^{\prime} B_{y}^{\prime}\right.
\end{array}\right),
$$

which is the same for both equations ( $i=1$ and $i=2$ ), and the vectors

$$
\mathbf{E}^{(i)}=\left(\begin{array}{c}
\left\langle\mathcal{E}_{i} B_{x}\right\rangle \\
\left\langle\mathcal{E}_{i} B_{y}\right\rangle \\
\left\langle\mathcal{E}_{i} B_{x}^{\prime}\right\rangle \\
\left\langle\mathcal{E}_{i} B_{y}^{\prime}\right.
\end{array}\right), \quad \mathbf{C}^{(i)}=\left(\begin{array}{c}
\alpha_{i x} \\
\alpha_{i y} \\
\eta_{i x z} \\
\eta_{i y z}
\end{array}\right) .
$$

The averages are taken over time. In Figs. 5 and 6 we show the results respectively for the coefficients $\alpha_{i j}$ and $\eta_{i j z}$ as functions of $z$. We also plot as a solid line a five point running mean of the data.

Here and below our error bars are a measure of the stability of the fit when only part of the time series is used. In practice we divide the data set into two parts and calculate the transport coefficients separately for each subset. The error bars cover then the range of values obtained by using all or only part of the data. The error bars would probably shrink if we extended the data set, but some of the noise is due to fluctuations from cycle to cycle and therefore physical.

Near the top boundary the data points deviate significantly from the relatively smooth trend seen in the data away from the boundary. Therefore, we consider the 

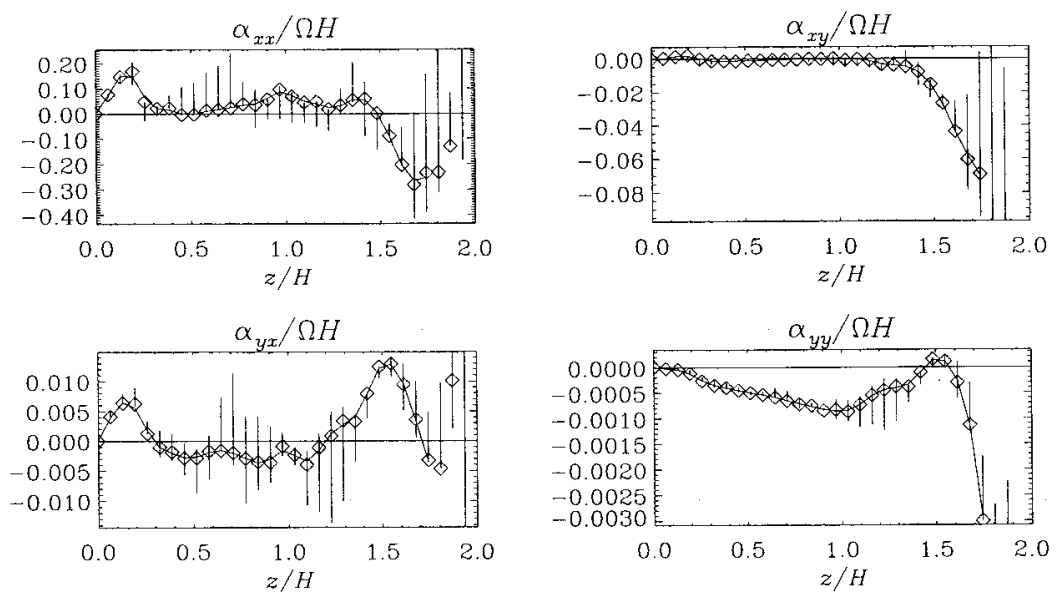

FIGURE 5 The symbols denote the results for $\alpha_{i j}(z)$ as obtained by solving (16) and the solid line represents a five point running mean.
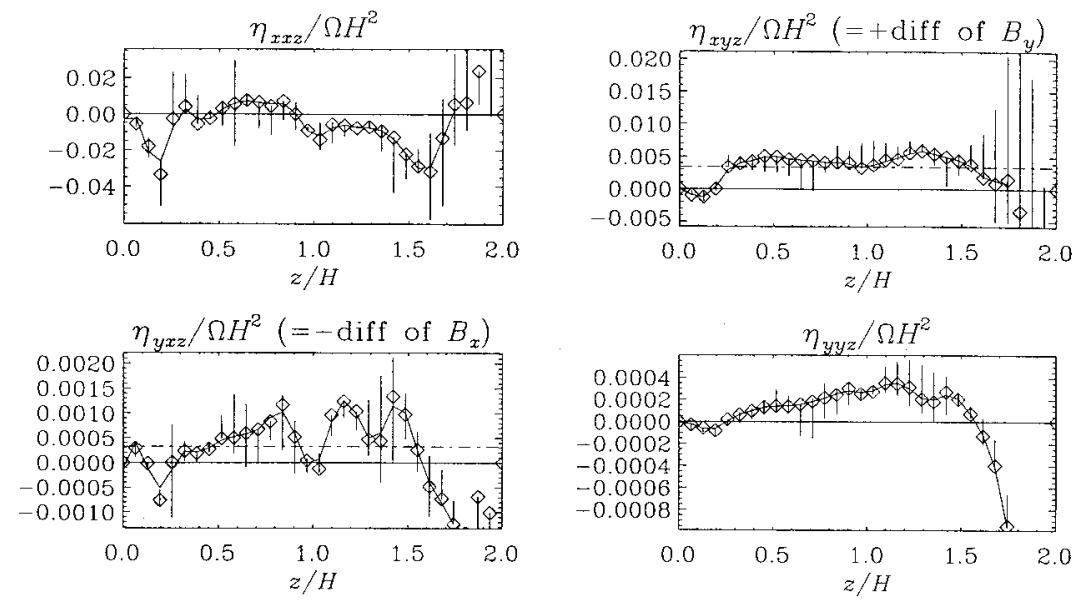

FIGURE 6 The symbols denote the results for $\eta_{i j z}(z)$ as obtained by solving (16) and the solid line represents a five point running mean. The two horizontal dash-dotted lines give the average values.

data near the boundary as uncertain and, when calculating averages and running means, we ignore all points within four mesh zones near the boundary. The vertically averaged values of the components of $\alpha_{i j}$ and $\eta_{i j z}$ are

$$
\alpha_{i j}=\left(\begin{array}{ll}
0.010 & -0.009 \\
0.001 & -0.001
\end{array}\right) \Omega H, \quad \eta_{i j z}=\left(\begin{array}{rr}
-0.007 & 0.003 \\
0.000 & 0.000
\end{array}\right) \Omega H^{2}
$$

Note that $\alpha_{y y}=-0.001 \Omega H$ and $\eta_{x y z}=0.003 \Omega H^{2}$, in rough agreement with the results found earlier from other considerations. However, $-\eta_{y x z}$ is at least ten times smaller and perhaps even negative. This coefficient is responsible for the diffusion of $B_{x}$, i.e., $\dot{B}_{x}=\cdots-\eta_{y x z} B_{x}^{\prime \prime}$. If $-\eta_{y x z}<0$, this would indicate that some higher order terms 
(hopefully with the right sign!) would need to be restored for stabilization. Typically, an infinite series of further terms could then become important (Dittrich et al., 1984; Elperin et al., 2000), making this whole approach difficult to use in practice. We address this difficulty in Sections 4.3 and 4.4 by adopting a nonlocal approach.

\subsection{A Local Formulation Using a Diagonal Diffusion Tensor}

We now adopt the $J$-formulation for the diffusion term, i.e. Eq. (3), using a diagonal diffusion tensor, i.e. $\eta_{x y}^{*}=\eta_{y x}^{*}=0$. In that case we have to solve two systems of three (instead of four) equations,

$$
\mathbf{E}^{(i)}(z)=\mathbf{M}^{(i)}(z) \mathbf{C}^{(i)}(z), \quad i=x, y,
$$

where

$$
\mathbf{M}^{(i)}=\left(\begin{array}{ccc}
\left\langle B_{x} B_{x}\right\rangle & \left\langle B_{x} B_{y}\right\rangle & -\left\langle B_{x} J_{i}\right\rangle \\
\left\langle B_{y} B_{x}\right\rangle & \left\langle B_{y} B_{y}\right\rangle & -\left\langle B_{y} J_{i}\right\rangle \\
-\left\langle J_{i} B_{x}\right\rangle & -\left\langle J_{i} B_{y}\right\rangle & \left\langle J_{i} J_{i}\right\rangle
\end{array}\right)
$$

is now different for the two equations $(i=1$ and $i=2$ ). (Here and below, no summation over $i$ is implied!) The vectors $\mathbf{E}^{(i)}$ are given by

$$
\mathbf{E}^{(i)}=\left(\begin{array}{c}
\left\langle\mathcal{E}_{i} B_{x}\right\rangle \\
\left\langle\mathcal{E}_{i} B_{y}\right\rangle \\
-\left\langle\mathcal{E}_{i} J_{i}\right\rangle
\end{array}\right), \quad \mathbf{C}^{(i)}=\left(\begin{array}{c}
\alpha_{i x} \\
\alpha_{i y} \\
\eta_{i i}^{*}
\end{array}\right) .
$$

Again, the averages are taken over time. In Figs. 7 and 8 we show the results respectively for the coefficients $\alpha_{i j}$ and $\eta_{i j}^{*}$ as functions of $z$. We also plot as a solid line a five point running mean of the data.
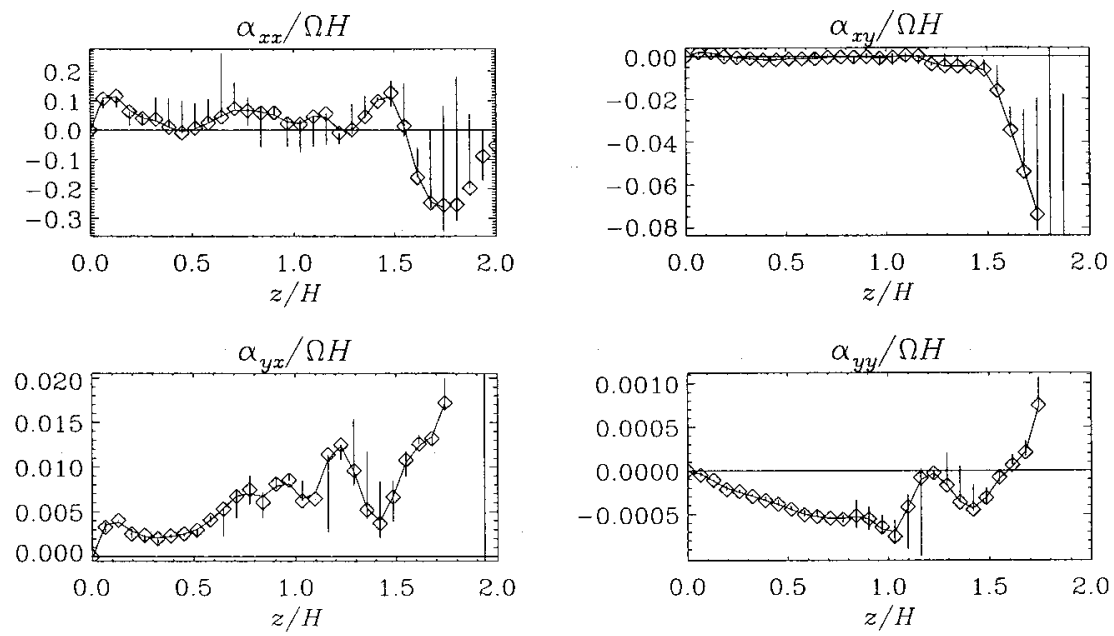

FIGURE 7 The symbols denote the results for $\alpha_{i j}(z)$ as obtained by solving (20) and the solid line represents a five point running mean. Here and below the error bars indicate the stability of the fit when only a subset of the data is used. 

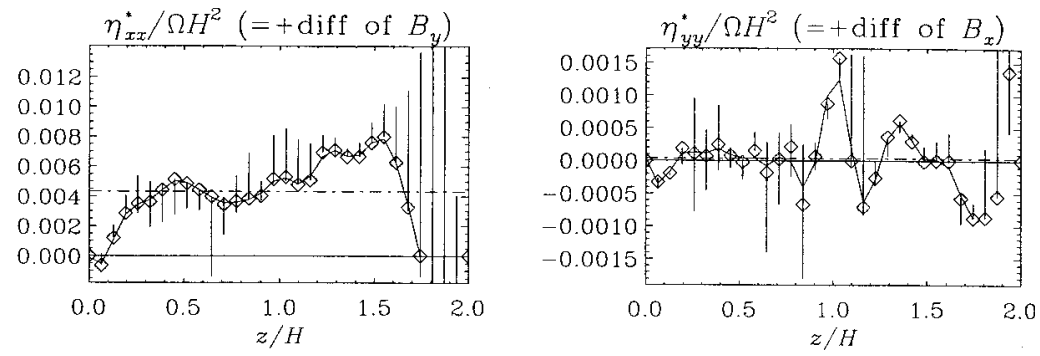

FIGURE 8 The symbols denote the results for $\eta_{i j}^{*}(z)$ as obtained by solving $(20)$ and the solid line represents a five point running mean. The average values are indicated by horizontal dash-dotted lines.

Note that both $\eta_{x x}^{*}$ and $\eta_{y y}^{*}$ are now positive on average (indicated by the dash-dotted line). Nevertheless, $\eta_{y y}^{*}$, which is responsible for the diffusion of $B_{x}$, shows still large fluctuations and can locally still be negative. Taken at face value, such excursions of $\eta_{y y}^{*}$ to negative values would cause a catastrophic growth of small-scale structures in a mean-field model. We note that the relation (4) between the different components of $\eta_{i j}^{*}$ and $\eta_{i j z}$ still holds approximately, even though this relation is no longer strictly valid if, as in the present case, the fit to the diagonal components of $\eta_{i j}^{*}$ is obtained under the restriction that the off-diagonal components vanish.

\subsection{A Nonlocal Formulation}

We recall that Eq. (2) corresponds really to a Taylor expansion of an underlying integral kernel in terms of derivatives of delta functions (see, e.g., Hasler et al., 1995; see also Nicklaus and Stix, 1988). Therefore, we now write Eqs. (14) and (15) as a convolution in the form

$$
\begin{aligned}
& \mathcal{E}_{x}=\alpha_{x x} * B_{x}+\alpha_{x y} * B_{y}+\eta_{x x z} * B_{x}^{\prime}+\eta_{x y z} * B_{y}^{\prime}, \\
& \mathcal{E}_{y}=\alpha_{y x} * B_{x}+\alpha_{y y} * B_{y}+\eta_{y x z} * B_{x}^{\prime}+\eta_{y y z} * B_{y}^{\prime},
\end{aligned}
$$

where $\alpha_{i j}\left(z, z^{\prime}, t\right)$ and $\eta_{i j z}\left(z, z^{\prime}, t\right)$ are now integral kernels and the asterisks refer to a convolution,

$$
\begin{gathered}
\alpha_{i j} * B_{j} \equiv \int_{0}^{L_{z}} \alpha_{i j}\left(z, z^{\prime}\right) B_{j}\left(z^{\prime}, t\right) d z^{\prime} \\
\eta_{i j z} * B_{j}^{\prime} \equiv \int_{0}^{L_{z}} \eta_{i j z}\left(z, z^{\prime}\right) B_{j}^{\prime}\left(z^{\prime}, t\right) d z^{\prime} .
\end{gathered}
$$

We solve for the 8 kernels using Fourier transformation. We introduce

$$
\hat{\mathcal{E}}_{i}^{\prime}=\int_{0}^{L_{z}} \mathcal{E}_{i}^{\prime}(z) \cos k z d z,
$$




$$
\hat{B}_{i}=\int_{0}^{L_{z}} B_{i}(z) \cos k z d z
$$

where

$$
k=k_{n}=(n+1 / 2) \pi / L_{z}, \quad n=0,1, \ldots
$$

Note that we use here the Fourier transform of the derivative of $\mathcal{E}$, which is more easily obtained from the simulations. We assume that $\alpha_{i j}\left(z, z^{\prime}\right)$ and $\eta_{i j z}\left(z, z^{\prime}\right)$ have the form

$$
\begin{gathered}
\alpha_{i j}\left(z, z^{\prime}\right)=\sin k_{0} z\left(2 / L_{z}\right) \sum_{k} \sin k z \sin k z^{\prime} \hat{\alpha}_{i j}(k), \\
\eta_{i j z}\left(z, z^{\prime}\right)=\left(2 / L_{z}\right) \sum_{k} \sin k z \sin k z^{\prime} \hat{\eta}_{i j z}(k),
\end{gathered}
$$

where $k_{0}=\pi /\left(2 L_{z}\right)$. We also need

$$
\tilde{B}_{i}(k)=\int_{0}^{L_{z}} B_{i}(z) \sin k_{0} z \sin k z d z,
$$

and the corresponding inverse transform in terms of $\tilde{B}_{i}(k)$, namely

$$
B_{i}(z)=\left(2 / L_{z}\right)\left(\sin k_{0} z\right)^{-1} \sum_{k} \sin k z \tilde{B}_{i}(k)
$$

The $\sin k_{0} z$ term in (30) ensures that the $\alpha$-effect is antisymmetric about the equatorial plane. For example, if $\hat{\alpha}_{i j}(k)$ is independent of $k$, then the integral in Eq. (25) simply becomes a multiplication, so $\alpha_{i j} * B_{j}=\sin k_{0} z \alpha_{i j}^{(0)} B_{j}(z)$, where $\alpha_{i j}^{(0)}=\hat{\alpha}_{i j}(k)$ is a constant, independent of $k$. We are now able to write (23) and (24) in the following form

$$
\begin{aligned}
& \hat{\mathcal{E}}_{x}^{\prime}=k \hat{\alpha}_{x x} \tilde{\boldsymbol{B}}_{x}+k \hat{\alpha}_{x y} \tilde{\boldsymbol{B}}_{y}-k^{2} \hat{\eta}_{x x z} \hat{\boldsymbol{B}}_{x}-k^{2} \hat{\eta}_{x y z} \hat{\boldsymbol{B}}_{y}, \\
& \hat{\mathcal{E}}_{y}^{\prime}=k \hat{\alpha}_{y x} \tilde{\boldsymbol{B}}_{x}+k \hat{\alpha}_{y y} \tilde{\boldsymbol{B}}_{y}-k^{2} \hat{\eta}_{y x z} \hat{\boldsymbol{B}}_{x}-k^{2} \hat{\eta}_{y y z} \hat{\boldsymbol{B}}_{y} .
\end{aligned}
$$

Again, we can solve for the 8 coefficients $\hat{\alpha}_{i j}$ and $\hat{\eta}_{i j z}$ by computing moments (separately for each value of $k$ ). We obtain two matrix equations of the form

$$
\tilde{\mathbf{E}}^{(i)}(k)=\tilde{\mathbf{M}}(k) \tilde{\mathbf{C}}^{(i)}(k), \quad i=x, y
$$

with the matrix

$$
\tilde{\mathbf{M}}=\left(\begin{array}{cccc}
\left\langle\tilde{\boldsymbol{B}}_{x} \tilde{\boldsymbol{B}}_{x}\right\rangle & \left\langle\tilde{\boldsymbol{B}}_{x} \tilde{\boldsymbol{B}}_{y}\right\rangle & \left\langle\tilde{\boldsymbol{B}}_{x} \hat{\boldsymbol{B}}_{x}\right\rangle & \left\langle\tilde{\boldsymbol{B}}_{x} \hat{\boldsymbol{B}}_{y}\right\rangle \\
\left\langle\tilde{\boldsymbol{B}}_{y} \tilde{\boldsymbol{B}}_{x}\right\rangle & \left\langle\tilde{\boldsymbol{B}}_{y} \tilde{\boldsymbol{B}}_{y}\right\rangle & \left\langle\tilde{\boldsymbol{B}}_{y} \hat{\boldsymbol{B}}_{x}\right\rangle & \left\langle\tilde{\boldsymbol{B}}_{y} \hat{\boldsymbol{B}}_{y}\right\rangle \\
\left\langle\hat{\boldsymbol{B}}_{x} \tilde{\boldsymbol{B}}_{x}\right\rangle & \left\langle\hat{\boldsymbol{B}}_{x} \tilde{\boldsymbol{B}}_{y}\right\rangle & \left\langle\hat{\boldsymbol{B}}_{x} \hat{\boldsymbol{B}}_{x}\right\rangle & \left\langle\hat{\boldsymbol{B}}_{x} \hat{\boldsymbol{B}}_{y}\right\rangle \\
\left\langle\hat{\boldsymbol{B}}_{y} \tilde{\boldsymbol{B}}_{x}\right\rangle & \left\langle\hat{\boldsymbol{B}}_{y} \tilde{\boldsymbol{B}}_{y}\right\rangle & \left\langle\hat{\boldsymbol{B}}_{y} \hat{\boldsymbol{B}}_{x}\right\rangle & \left\langle\hat{\boldsymbol{B}}_{y} \hat{\boldsymbol{B}}_{y}\right\rangle
\end{array}\right),
$$


which is the same for both equations $(i=1$ and $i=2)$, and the vectors

$$
\tilde{\mathbf{E}}^{(i)}=\left(\begin{array}{c}
\left\langle\hat{\mathcal{E}}_{i}^{\prime} \tilde{\boldsymbol{B}}_{x}\right\rangle \\
\left\langle\hat{\mathcal{E}}_{i}^{\prime} \tilde{\boldsymbol{B}}_{y}\right\rangle \\
\left.\hat{\mathcal{E}}_{i}^{\prime} \hat{\boldsymbol{B}}_{x}\right\rangle \\
\left\langle\hat{\mathcal{E}}_{i}^{\prime} \hat{\boldsymbol{B}}_{y}\right\rangle
\end{array}\right), \quad \tilde{\mathbf{C}}^{(i)}=\left(\begin{array}{c}
k \hat{\alpha}_{i x} \\
k \hat{\alpha}_{i y} \\
-k^{2} \hat{\eta}_{i x z} \\
-k^{2} \hat{\eta}_{i y z}
\end{array}\right) .
$$

The averages are taken over time. In Figs. 9 and 10 we show the results respectively for the coefficients $\hat{\alpha}_{i j}$ and $\hat{\eta}_{i j z}$ as functions of $k$. We also plot as a solid line a five point
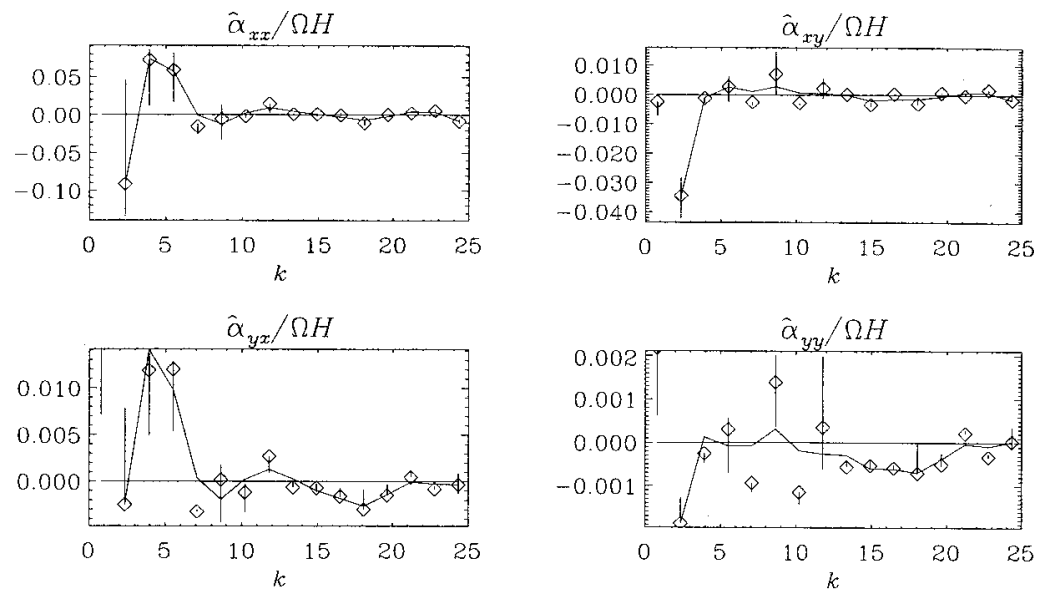

FIGURE 9 The symbols denote the results for $\hat{\alpha}_{i j}(k)$ as obtained from (36) and the solid line represents a five point running mean ignoring the point $k=k_{0}$.
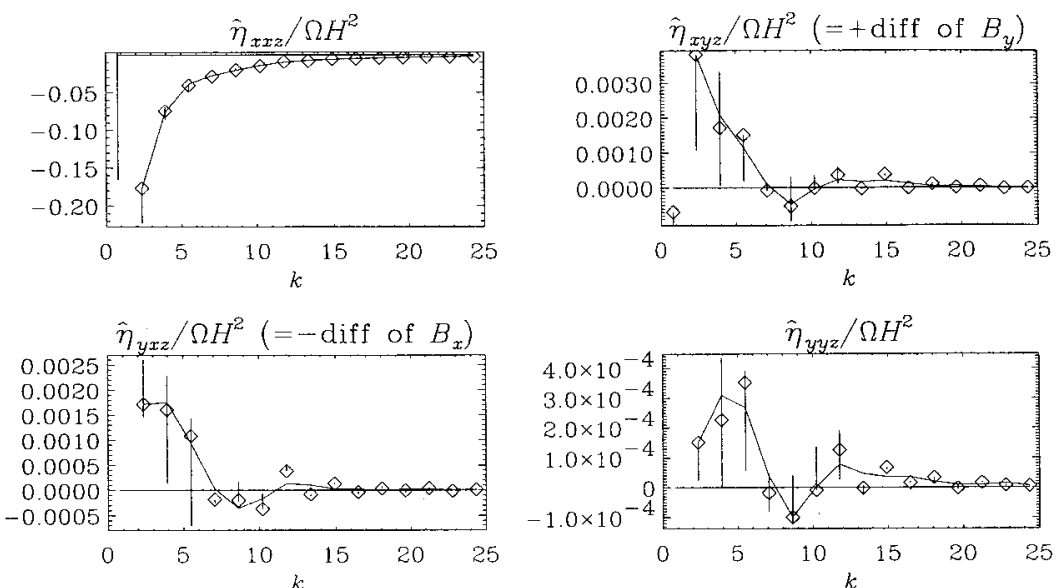

FIGURE 10 The symbols denote the results for $\hat{\eta}_{i j z}(k)$ as obtained from (36) and the solid line represents a five point running mean. Note that the data points for the smallest value of $k, k=k_{0}$, deviate strongly from those for $k_{1}$ and lie outside the plot $\left(\hat{\eta}_{x x z}\left(k_{0}\right)=0.13 \Omega H^{2}, \hat{\eta}_{x y z}\left(k_{0}\right)=-0.0007 \Omega H^{2}, \hat{\eta}_{y x z}\left(k_{0}\right)=0.04 \Omega H^{2}\right.$ and $\left.\hat{\eta}_{y y}^{*}\left(k_{0}\right)=0.002 \Omega H^{2}\right)$. 
running mean of the data. On the boundaries the lines go through the original data points, however.

In the traditional $\alpha \Omega$-dynamo the $(y, y)$-component is the most important one. However, $\hat{\alpha}_{y y}(k)$ shows substantial variations in $k$, indicating possibly large uncertainties, although $\hat{\alpha}_{y y}$ is mostly negative and the value for $k_{1}$ is around -0.001 , similar to the value we used in Eq. (13). For the diffusion coefficient $\hat{\eta}_{x y z}$ the value for $k=k_{1}$ is positive, i.e. stabilizing. Furthermore, the value for $k=k_{1}$ is much larger than for the next higher values of $k$. One possible suggestion would be to assume that $\eta_{i j z}(k)$ was proportional to $k^{-2}$. However, this would correspond to diffusion of the algebraic form $\dot{B}_{i}=\ldots-B_{i} / \tau^{(i)}$, i.e. without any differential (or integral) operator. Here the superscript $(i)$ on $\tau$ indicates that the decay times are in general different for the $x$ and $y$ directions. Let us recall, however, that because the eigenvalues of the diffusion tensor are not all positive our fitting is still not self-consistent.

\subsection{A Nonlocal Formulation Using a Diagonal Diffusion Tensor}

Like in Section 4.2 we now adopt the formulation (3) using a diagonal diffusion tensor. We write

$$
\begin{aligned}
& \hat{\mathcal{E}}_{x}^{\prime}=k \hat{\alpha}_{x x} \tilde{B}_{x}+k \hat{\alpha}_{x y} \tilde{B}_{y}-\hat{\eta}_{x x}^{*} \hat{J}_{x}^{\prime}, \\
& \hat{\mathcal{E}}_{y}^{\prime}=k \hat{\alpha}_{y x} \tilde{B}_{x}+k \hat{\alpha}_{y y} \tilde{B}_{y}-\hat{\eta}_{y y}^{*} \hat{\boldsymbol{J}}_{y}^{\prime},
\end{aligned}
$$

where $\hat{\boldsymbol{J}}_{x}^{\prime}=+k^{2} \hat{\boldsymbol{B}}_{y}$ and $\hat{\boldsymbol{J}}_{y}^{\prime}=-k^{2} \hat{\boldsymbol{B}}_{x}$. Instead of Eq. (36) we have

$$
\tilde{\mathbf{E}}^{(i)}(k)=\tilde{\mathbf{M}}^{(i)}(k) \tilde{\mathbf{C}}^{(i)}(k), \quad i=x, y
$$

with the matrix

$$
\tilde{\mathbf{M}}^{(i)}=\left(\begin{array}{ccc}
\left\langle\tilde{B}_{x} \tilde{\boldsymbol{B}}_{x}\right\rangle & \left\langle\tilde{\boldsymbol{B}}_{x} \tilde{\boldsymbol{B}}_{y}\right\rangle & -\left\langle\tilde{\boldsymbol{B}}_{x} \hat{\boldsymbol{J}}_{i}^{\prime}\right\rangle \\
\left\langle\tilde{\boldsymbol{B}}_{y} \tilde{\boldsymbol{B}}_{x}\right\rangle & \left\langle\tilde{\boldsymbol{B}}_{y} \tilde{\boldsymbol{B}}_{y}\right\rangle & -\left\langle\tilde{\boldsymbol{B}}_{y} \hat{\boldsymbol{J}}_{i}^{\prime}\right\rangle \\
-\left\langle\hat{\boldsymbol{J}}_{i}^{\prime} \tilde{\boldsymbol{B}}_{x}\right\rangle & -\left\langle\hat{\boldsymbol{J}}_{i}^{\prime} \tilde{\boldsymbol{B}}_{y}\right\rangle & \left\langle\hat{\boldsymbol{J}}_{i}^{\prime} \hat{\boldsymbol{J}}_{i}^{\prime}\right\rangle .
\end{array}\right) .
$$

(Again, here and below no summation over $i$ is implied!) The vectors are

$$
\tilde{\mathbf{E}}^{(i)}=\left(\begin{array}{c}
\left\langle\hat{\mathcal{E}}^{\prime} \tilde{B}_{x}\right\rangle \\
\left\langle\hat{\mathcal{E}}_{i}^{\prime} \tilde{B}_{y}\right\rangle \\
-\left\langle\hat{\mathcal{E}}_{i}^{\prime} \hat{J}_{i}^{\prime}\right\rangle
\end{array}\right), \quad \tilde{\mathbf{C}}^{(i)}=\left(\begin{array}{c}
k \hat{\alpha}_{i x} \\
k \hat{\alpha}_{i y} \\
\hat{\eta}_{i i}^{*}
\end{array}\right) .
$$

In Figs. 11 and 12 we show the results respectively for the coefficients $\hat{\alpha}_{i j}$ and $\hat{\eta}_{i j}^{*}$ as functions of $k$. We also plot as a solid line a five point running mean of the data.

Now both $\hat{\eta}_{x x}^{*}(k)$ and $\hat{\eta}_{y y}^{*}(k)$ are positive in the range $1 \lesssim k \lesssim 15$. Like in Section 4.2 the restriction to a purely diagonal magnetic diffusion tensor has helped to make turbulent diffusion positive definite. For larger values of $k$, corresponding to smaller scales, the data are probably no longer reliable. For the smallest value of $k, k=k_{0}$, the diffusion coefficient of $B_{y}$ is negative, corresponding to a destabilization of the field on large scales. 

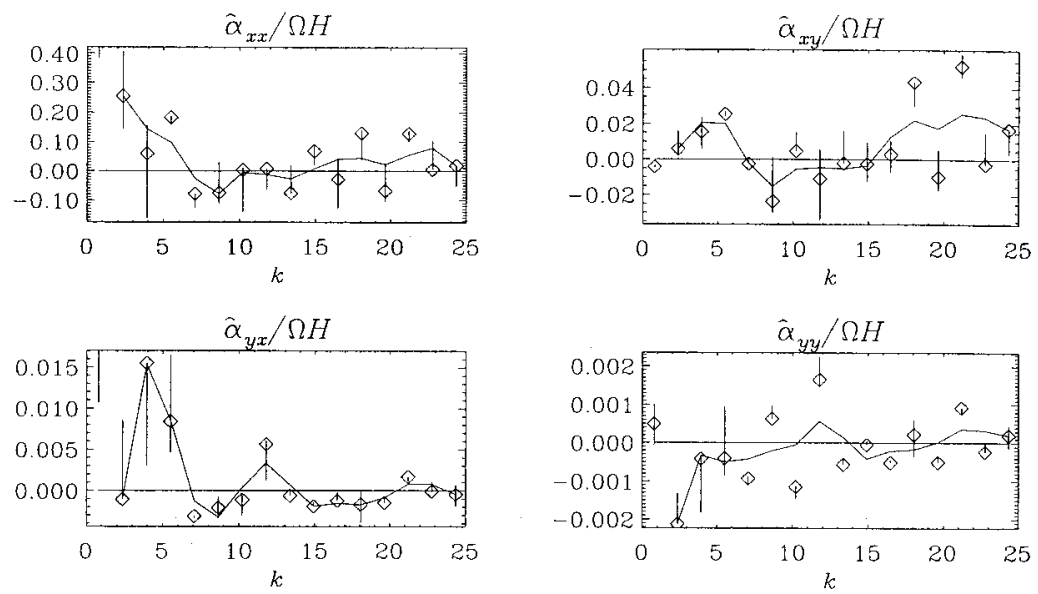

FIGURE 11 The symbols denote the results for $\hat{\alpha}_{i j}(k)$ as obtained from (41)and the solid line represents a five point running mean ignoring the point $k=k_{0}$.
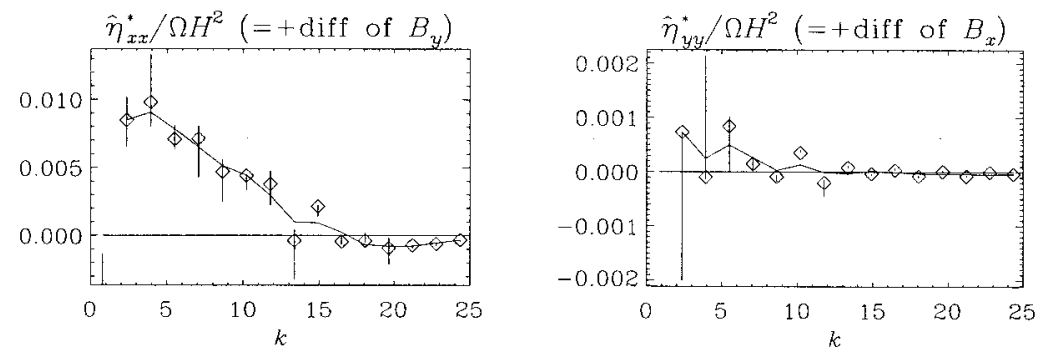

FIGURE 12 The symbols denote the results for $\hat{\eta}_{i j}^{*}(k)$ as obtained from (41) and the solid line represents a five point running mean. Note that the data points for the smallest value of $k, k=k_{0}$, deviate strongly from those for $k_{1}$ and lie outside the plot $\left(\hat{\eta}_{x x}^{*}\left(k_{0}\right)=-0.004 \Omega H^{2}\right.$ and $\left.\hat{\eta}_{y y}^{*}\left(k_{0}\right)=0.01 \Omega H^{2}\right)$.

While this is of course what is seen in the simulation, it is unclear whether this is really due to a negative diffusion on large scales (like in the Kuramoto-Sivashinsky equation with negative diffusion and positive hyperdiffusion), or just a natural manifestation of the $\alpha$-effect or an inverse cascade. Let us note that the negative diffusion in the largest scale does not lead to any catastrophic behaviour of the solution. For recent work on the negative diffusion effect see Zheligovsky et al. (2001).

\subsection{Comparative Remarks}

In the previous subsections we have seen that $\alpha$-effect and turbulent diffusion are not only always tensors, but their components show also systematic spatial variations and, more importantly, they are dominated by only the smallest wavenumbers. It does not seem feasible, however, to determine simultaneously spatial variations and wavenumber dependence. We emphasize that we do not advocate that the model of Section 4.4 is better than that of Section 4.2 or even Section 3, for example. Instead, elements of both approaches should preferentially be taken into consideration. 
Generally, we do find, however, that the restriction to only diagonal components of the $\eta_{i j}^{*}(z)$ or $\hat{\eta}_{i j}^{*}(k)$ tensors is to be preferred. This is because, on physical grounds, these diagonal components should be positive. If one does allow for off-diagonal components, as in Sections 4.1 and 4.3, one finds (as expected) somewhat different results $\left(-\eta_{y x z}\right.$ is different from $\eta_{y y}^{*}$, for example) and diffusion of $B_{x}$ field is then mostly negative.

We wish to emphasize the importance of simultaneously determining the various transport coefficients, as we have attempted here. To our knowledge, the only other time this was done was in B01 where scalar $\alpha$ and $\eta_{\mathrm{t}}$ quenchings have been determined. In $\mathrm{B} 01$, both direct determination and fitting to a model were used to obtain transport coefficients. Of course, fitting to a model is a much more stable and reliable procedure, but it is necessarily model dependent and one still has to assess whether or not the model is actually consistent with the data.

An important complication arises from the fact that the actual electromotive force contains a strongly fluctuating component which is physical. This affects the results obtained from a direct determination of transport coefficients and, consequently, many of the detailed features cannot be physically meaningful. To our knowledge, dynamo models with nonlocal (wavenumber dependent) transport coefficients were never considered before. In order to have a preliminary assessment of such models, and to obtain insight as to which components are crucial, we now consider simple model calculations with wavenumber dependent transport coefficients.

\section{MODEL CALCULATIONS}

The direct determination of nonlocal transport coefficients used in Section 4 suggests that nonlocal effects could be important. In fact, the magnitudes of $\hat{\alpha}_{i j}$ and $\hat{\eta}_{i j}^{*}$ tend to decrease with $k$. A $k^{-2}$ dependence of $\hat{\eta}_{x x}^{*}$ and $\hat{\eta}_{y y}^{*}$ would cancel the $\nabla^{2}$ diffusion operator and would yield a simple (and again local!) $-1 / \tau$ damping term. The problem with this type of damping is clear when looking at the dispersion relation (10). For simplicity we assume a scalar magnetic diffusivity, $\hat{\eta}_{x x}^{*}(k)=\hat{\eta}_{y y}^{*}(k) \equiv \hat{\eta}_{\mathrm{t}}(k)$, and that $\hat{\eta}_{\mathrm{t}}(k)=\left(\tau k^{2}\right)^{-1}$ we have

$$
\lambda(k)=(1 \pm i)\left|\frac{3}{4} \Omega \hat{\alpha}_{y y}(k) k\right|^{1 / 2}-\tau^{-1},
$$

so for $\hat{\alpha}_{y y}(k)=$ const, we would have $\lambda(k)>0$ for some $k>k_{\text {crit }}$. In order to prevent such an 'ultraviolet catastrophe' we have to require that $\hat{\alpha}_{y y}(k) \rightarrow 0$ for large values of $k$. On the other hand, the product $\hat{\alpha}_{y y}(k) k$ should reach its maximum for relatively small values of $k$, because the simulations (Fig. 1) suggested no systematic high- $k$ dependence.

Looking at Fig. 11, $\hat{\alpha}_{y y}(k)$ fluctuates essentially about zero, although it does show a negative peak at $k=3 \pi / 4 \approx 2.4$. Although $\left|\hat{\alpha}_{x x}\right|$ is much larger than $\left|\hat{\alpha}_{y y}\right|$, it is relatively unimportant because it multiplies $B_{x}$, which is small, and $\hat{\alpha}_{x x}$ only contributes to generating $B_{y}$ field, which is more effectively generated by the shear term. Given that it is only $\hat{\alpha}_{y y}(k)$ which contributes to $\alpha \Omega$-type dynamo action, we need to model this term somehow. We do this by assuming a linear dependence on $k$ for $k \leq 4$ of the form

$$
\hat{\alpha}_{y y}(k)=-0.001\left(1-k / k_{\max }\right) \quad \text { for } k \leq k_{\max }=4 \text {. }
$$



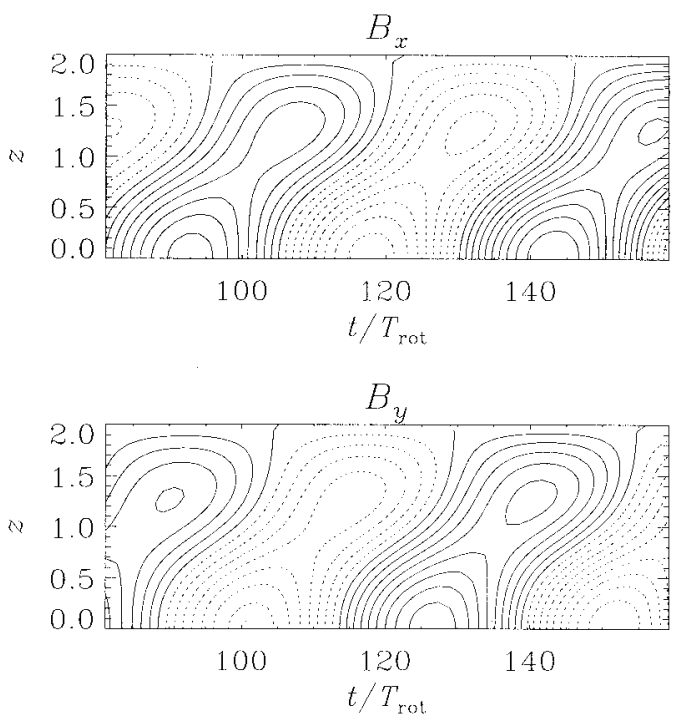

FIGURE 13 Model with $\hat{\alpha}_{y y}(k)=-0.001\left(1-k / k_{\max }\right), k \leq k_{\max }=4, \tau^{-1}=0.01$.

For $\hat{\eta}_{\mathrm{t}}(k)$ we assume

$$
\hat{\eta}_{\mathrm{t}}(k)=\left(\tau k^{2}\right)^{-1} \quad \text { with } \tau^{-1}=0.01
$$

The resulting butterfly diagram is quite reasonable; see Fig. 13. The main difference compared with the local model shown in Fig. 3 is that the dynamo wave migrates now slower. The fast migration was the main shortcoming of the local model, where field reversals happened almost simultaneously both at the midplane and at the upper boundary.

The assumption of a scalar $\hat{\eta}_{\mathrm{t}}(k)$ is made purely for simplicity. For comparison we have also considered a model with $\hat{\eta}_{y y}^{*}(k)=0.1 \hat{\eta}_{x x}^{*}(k)$ (as suggested by Fig. 12) and $\hat{\eta}_{x x}^{*}(k)=\left(\tau k^{2}\right)^{-1}$ with $\tau^{-1}=0.01$ (as before). In order that the model is still only marginally excited, we had to reduce $\hat{\alpha}_{y y}(k)$ by a uniform scaling factor of 5 . Except for an increase of the cycle period by about a factor of 2 , the resulting butterfly diagram was almost the same as in (Fig. 13).

Given that (i) this approximation reproduces phenomenology, (ii) is based on a fit to the actual electromotive force (see Figs. 11 and 12), and (iii) the dissipation is positive definite, we consider Eqs. (45) and (46) as the most reasonable parameterization if one wanted to use mean-field theory.

The results for many of the components of the $\alpha$ - and $\eta$-tensors are rather noisy. However, the fact that the obtained values for $\alpha_{y y}, \hat{\alpha}_{y y}(k)$, and $\eta_{\mathrm{t}}$ and $\left(\tau k^{2}\right)^{-1}$, are all of the same sign and of comparable magnitude suggests this is a stable result of the analysis. This result is also in agreement with what is required for a mean-field model to work. Firstly, the sign of $\hat{\alpha}_{y y}$ has to be negative to make the dynamo wave move with a positive migration speed. Secondly, the magnitude of $\hat{\alpha}_{y y}$ has to be around $10^{-3}$ so that the dynamo period is around 30 rotational periods. Finally, $\eta_{\mathrm{t}}$ has to be around $10^{-2}$ so that the dynamo is just weakly supercritical. 


\section{NOISE IN THE TRANSPORT COEFFICIENTS}

The plots for both local and nonlocal approaches show strong fluctuations in the various transport coefficients. This is caused by strong noise, which is much more pronounced in the electromotive force than in the resulting mean field. It would therefore be surprising if the $\alpha$-effect was really responsible for generating the much less noisy large scale magnetic field in the simulation. However, it is important to note that even a completely noisy $\alpha$-effect with no net component can amplify magnetic fields (Moffatt, 1978). Furthermore, in the presence of large scale shear it is possible to generate also a large scale field (Vishniac and Brandenburg, 1997).

Given the high level of noise found in the transport coefficients it is useful to elaborate further on this point. In the following we present a model calculation where the effect of strong noise is included. We solve Eqs. (5)-(9) and replace Eq. (13) by

$$
\alpha_{y y}=-0.001 \Omega z+\alpha_{N} N, \quad \eta_{\mathrm{t}}=0.0055 \Omega H^{2},
$$

where $\alpha_{N}$ is a coefficient and $N(z, t)$ is gaussian noise in $z$ and $t$, normalized to a root-mean-square value of unity. The other components of $\alpha_{i j}$ and $\eta_{i j}^{*}$ are ignored because we want to examine the effect of noise and take therefore the simplest possible model that was presented already in Fig. 3. In Fig. 14 we present the result for $\alpha_{N}=0.01$, corresponding to a noise level that exceeds the mean $\alpha$-effect by a factor ten or more, especially close to the midplane, where the coherent $\alpha$-effect vanishes.

The resulting field structure looks rather similar to the mean field obtained by averaging the data from the original three-dimensional simulations. The noise level exceeds the mean (coherent) $\alpha$-effect by more than a factor of ten, which is comparable to or even larger than what has been suggested in connection with the solar dynamo (Choudhuri, 1992; Moss et al., 1992; Hoyng et al., 1994; Otmianowska-Mazur,
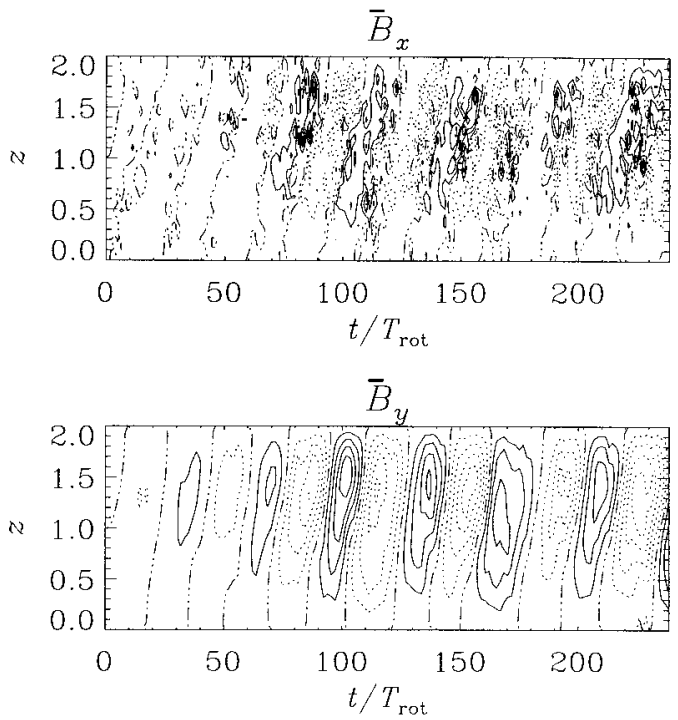

FIGURE 14 Mean-field calculation with $\alpha_{N}=0.01, \alpha_{0}=-0.001$, and $\eta_{0}=0.005$. 
1997). The main point we want to make here is that a high noise level in the mean-field transport coefficients (Section 4) is quite natural and not simply an indication of inaccurate measurements.

\section{COMMENTS ON NONLINEAR QUENCHING}

The issue of magnetic quenching of turbulent transport coefficients has not been addressed in the present article. One must expect that all components of the $\alpha$ and $\eta$ tensors depend on magnetic field strength and field structure. In principle our approach could be modified to allow for such nonlinearities. The usual assumption is that, if the turbulent transport coefficients are caused by turbulent convection, for example, $\alpha$ and $\eta_{\mathrm{t}}$ would decrease with increasing field strength. On the other hand, if the turbulent transport coefficients are caused by flow fields which themselves are magnetically driven, for example by magnetic (e.g., Balbus-Hawley and Parker) instabilities, then $\alpha$ and $\eta_{\mathrm{t}}$ may actually increase with increasing field strength (see Brandenburg, 1997 for a recent discussion on that). There is even some observational evidence for this somewhat unusual proposal (Brandenburg et al., 1998; Saar and Brandenburg, 1999). Thus, in addition to allowing $\alpha$ and $\eta_{\mathrm{t}}$ to be scale dependent, one should also allow for the possibility that $\alpha$ and $\eta_{\mathrm{t}}$ may actually increase with magnetic field strength. However, these type of behaviours must still be compatible with helicity conservation.

In the case of a fully periodic domain the situation is now fairly well understood. For example, we know that the correct helicity limited growth of the large scale field can be reproduced if both $\alpha$ and $\eta_{\mathrm{t}}$ are equally strongly quenched by the mean magnetic field, $\overline{\mathbf{B}}$, and the quenching is of the form $1 /\left(1+\alpha_{B}|\overline{\mathbf{B}}|^{2} / B_{\text {eq }}^{2}\right)$, where $B_{\text {eq }}$ is the equipartition field strength, and the coefficient $\alpha_{B}$ is proportional to the magnetic Reynolds number. This type of 'catastrophic' quenching goes back to the early article by Vainshtein and Cattaneo (1992), and was confirmed using simulations (Cattaneo and Hughes, 1996), but it is now clear that this behaviour is primarily a consequence of magnetic helicity conservation (Blackman and Field, 2000; B01), and independent of the type of nonlinearity (Brandenburg and Subramanian, 2000). Evidence for 'catastrophic' $\eta_{\mathrm{t}}$-quenching was first found by Cattaneo and Vainshtein (1991) using two-dimensional simulations. Three-dimensional simulations of isotropic helical turbulence in a box yielded a resistively slow saturation behavior of $\alpha$ which is well described by a fit of the form $|\overline{\mathbf{B}}|^{2} \sim 1-\exp \left(-2 \eta k^{2} \Delta t\right)$, where $\eta$ is the microscopic magnetic diffusivity, $k$ is the wavenumber of the large scale field, and $\Delta t$ is the time after which the small scale field has saturated. The necessity to obey this fit is sufficiently stringent to exclude for example cubic quenching behaviour (e.g., Moffatt, 1972; Rüdiger, 1974; Rüdiger and Kitchatinov, 1993), or quenching of the form $1-\alpha_{B}|\overline{\mathbf{B}}|^{2} / B_{\text {eq }}^{2}$. As was recently pointed out by Field and Blackman (2002), a type of quenching that satisfies the magnetic helicity equation exactly and that is therefore also compatible with resistively limited growth is dynamical quenching (Kleeorin et al., 1995). According to this description, $\alpha$ and $\eta_{\mathrm{t}}$ are no longer catastrophically quenched. In other words, the earlier conclusion of B01 is not compulsory because of a degeneracy of the dynamo equations in the fully helical case.

In a non-periodic domain the situation is quite different, because there is then the possibility of a magnetic helicity flux out of the domain. This magnetic helicity loss is associated with a loss of magnetic energy. In the model of BD much of the field 
that was lost through the boundaries was of large scale, which made the large scale dynamo process less efficient. This corresponds to a less drastic quenching, because $\alpha_{B}$ is now only proportional to the square root of the magnetic Reynolds number (BD).

The presence of shear affects the saturation results in a different way. Field amplification by shear is quite independent of the magnetic helicity effect, but this concerns only the toroidal field amplification. For the dynamo to work one still needs to generate poloidal field in order to make use of the shear. Thus, saturation of the large scale field still occurs on a resistive time scale, but larger field amplitudes are now possible (Brandenburg et al., 2001). Because of shear, the field evolves in an oscillatory fashion (as predicted by mean-field $\alpha \Omega$-dynamo theory; e.g. Moffatt, 1978; Parker, 1979). It is at present not entirely clear, however, whether the time scale for this oscillation is resistive or dynamical.

\section{CONCLUSIONS}

In the present article we have demonstrated that the results of the three-dimensional numerical nonlinear dynamo simulations of BNST95 can be fitted by a mean-field dynamo model with properly chosen parameterizations of transport coefficients. The parameterization found from such a fitting procedure is far from that of naive kinematic mean-field dynamo theory. In fact, our investigations point toward the possibility that turbulent transport coefficients might be wavenumber dependent. This possibility was first explored by Moffatt (1983), who used a renormalization group approach to obtain differential equations for $\alpha$ and $\eta_{\mathrm{t}}$ as functions of wavenumber. We find that the strongest contribution to $\alpha$ and $\eta_{\mathrm{t}}$ tends to come from the largest scales in the system. Unfortunately the scatter in the data is considerable and only data for the first few wavenumbers seem to be significant. However, simple representations of the form (45) and (46) seem to reproduce the original data reasonably well (compare Fig. 13 with Fig. 1). These representations are consistent with fits to the electromotive force and, more importantly, the corresponding energy dissipation is now positive.

Note that we have restricted the diffusion tensor that multiplies the current to be diagonal. This restriction means that the turbulent diffusion does not mix $x$ and $y$ components of the mean magnetic field. Such kind of 'cross-talk' is absent in dynamo models with short-correlated random velocity fields (Molchanov et al., 1985), although it may exist in other models (Rädler's $\Omega \times J$-effect is an example; see Krause and Rädler, 1980). We note that our estimates for the $\alpha$-tensor are not significantly modified when the diffusion tensor is restricted to be diagonal.

Comparing the results of Sections 3 and 4.1-4.4, several similar aspects can be recovered in each case. Firstly, $\alpha_{y y}$ is negative in the upper hemisphere (or disc plane) and its magnitude, in natural units, is around $10^{-3}$ at $z \approx H$ (see Figs. 5 and 7 ) or at small wave numbers $\left(k=k_{1}\right)$, i.e. at large scales (see Figs. 9 and 11). Secondly, the turbulent magnetic diffusivity in the streamwise direction $(y)$ is such that the corresponding mean-field dynamo is marginally excited. The turbulent magnetic diffusivity in the cross-stream direction $(x)$ is however much smaller than in the streamwise direction (cf. the two panels in Fig. 8). This is again reflected in the nonlocal approach (cf. the two panels in Fig. 12), but here the diffusion coefficient of $B_{x}$ is more noisy and, at the minimum wavenumber $k_{0}$, the diffusion coefficient of $B_{x}$ becomes comparable to that of $B_{y}$, but it stays at least positive. We regard similarities in the results of the 
various approaches as an indication of their robustness (e.g., the values of $\alpha_{y y}$ and $\eta_{x x}^{*}$ ). On the other hand, dissimilarities and noisy results, as well as large error bars, indicate that results are not reliable (e.g. $\eta_{y y}^{*}$ and $\hat{\eta}_{y y}^{*}$ ). Positivity of the diffusion coefficients is an additional criterion for reliability.

We should stress that we expect realistic transport coefficients to show both height dependence as well as scale dependence. In addition, the $\alpha$-effect is a dynamical one that satisfies an explicitly time-dependent equation (Rogachevskii and Kleeorin, 2001). It is however rather difficult to determine all these aspects simultaneously, which is why we have looked at each of them separately. Indeed, our goal is therefore not to distinguish between the various approaches, but rather to present a first assessment of the presence of each of these effects (height dependence, scale dependence), all of which should be present simultaneously.

We stress that our final model is based on very noisy fits for $\alpha_{y y}$. We feel that it is important to acknowledge the reality and physical significance of unsteady and random transport coefficients and to investigate models with stochastic alpha. Note also that there are some models showing the possibility of the generation of large scale magnetic patterns in a flow with a random (incoherent) $\alpha$-effect (Vishniac and Brandenburg, 1997). However, in order to reproduce the butterfly (space-time) diagram obtained from the three-dimensional simulations a coherent (non-noisy) $\alpha$-effect must still be present. The present results suggest that the simulations are reproduced with a coherent $\alpha$-effect that is only about $10 \%$ of the incoherent $\alpha$-effect in parts of the domain.

The idea that only the smallest wavenumbers contribute to the transport coefficients may well prove to be a reasonable representation for astrophysical dynamos. Already now there is some evidence for this proposal: so far only models that include the lowest wavenumber are able to reproduce stellar cycle frequencies that decrease sufficiently rapidly with increasing rotational frequency (Brandenburg et al., 1998). By contrast, models with transport coefficients that are independent of wavenumber (Tobias, 1998) show that the cycle frequency decreases too slowly with increasing rotational frequency.

Although the present work was motivated by astrophysical and geophysical applications, the analysis presented here may just as well apply to homogeneous dynamos in the laboratory such as the Karlsruhe experiment (Stieglitz and Müller, 2001). The results from these experiments can now accurately be tested against simulations (Tilgner, 2000) and mean-field theory (Rädler et al., 1998). Due to the presence of boundaries, however, the $\alpha$ and $\eta$ tensors have to be replaced by integral kernels (Stefani et al., 2000). One may therefore, expect that the approach explored in the present article to determine integral kernels for the $\alpha$ and $\eta$ tensors will soon gain in importance.

\section{Acknowledgements}

We thank David Moss and Günther Rüdiger for helpful suggestions that have helped to improve the presentation of the article. One of us (DS) is grateful for financial support by the Russian Foundation for Basic Research under grant 99-01-00362, the NATO Collaborative Research Grant PST.CLG 974737, as well as the Royal Society for an ex-quota grant, which enabled him to spend half a year in Newcastle, where most of this work was carried out. 


\section{References}

Arlt, R. and Brandenburg, A. "Search for non-helical disc dynamos in simulations", Astron. Astrophys., 380 , 359-372 (2001).

Balbus, S.A. and Hawley, J.F. “A powerful local shear instability in weakly magnetized disks. I. Linear analysis", Astrophys. J. 376, 214-222 (1991).

Blackman, E.G. and Field, G.F. "Constraints on the magnitude of $\alpha$ in dynamo theory", Astrophys. J. 534, 984-988 (2000).

Brandenburg, A. "Large scale turbulent dynamos", Acta Astron. Geophys. Univ. Comenianae XIX, 235-261 (1997).

Brandenburg, A. "Disc turbulence and viscosity". In: Theory of Black Hole Accretion Discs (Eds. M.A. Abramowicz, G. Björnsson and J.E. Pringle) pp. 61-86, Cambridge University Press (1998).

Brandenburg, A. "The inverse cascade and nonlinear alpha-effect in simulations of isotropic helical hydromagnetic turbulence", Astrophys. J. 550, 824-840, (2001). (B01).

Brandenburg, A., Bigazzi, A. and Subramanian, K. "The helicity constraint in turbulent dynamos with shear", Mon. Not. Roy. Astro. Soc. 325, 685-692, (2001).

Brandenburg, A. and Dobler, W. "Large scale dynamos with helicity loss through boundaries", Astron. Astrophys. 369, 329-338. (2001). (BD).

Brandenburg, A. and Donner, K.J. "The dependence of the dynamo alpha on vorticity", Mon. Not. Roy. Astro. Soc. 288, L29-L33 (1997).

Brandenburg, A. and Sarson, G.R. "The effect of hyperdiffusivity on turbulent dynamos with helicity", Phys. Rev. Lett. 88, 055003-1-4 (2002).

Brandenburg, A. and Subramanian, K. "Large scale dynamos with ambipolar diffusion nonlinearity", Astron. Astrophys. 361, L33-L36 (2000).

Brandenburg, A., Saar, S.H. and Turpin, C.R. "Time evolution of the magnetic activity cycle period", Astrophys. J. Lett. 498, L51-L54 (1998).

Brandenburg, A., Nordlund, A., Stein, R.F. and Torkelsson, U. "Dynamo generated turbulence and large scale magnetic fields in a Keplerian shear flow”, Astrophys. J. 446, 741-754 (1995). (BNST95).

Brandenburg, A., Nordlund, A., Stein, R.F. and Torkelsson, U. "The disk accretion rate for dynamo generated turbulence", Astrophys. J. Lett. 458, L45-L48 (1996). (BNST96).

Brandenburg, A., Nordlund, A., Pulkkinen, P., Stein, R.F. and Tuominen, I. "3-d simulation of turbulent cyclonic magneto-convection", Astron. Astrophys. 232, 277-291 (1990).

Cattaneo, F. and Hughes, D.W. "Nonlinear saturation of the turbulent alpha effect", Phys. Rev. E 54, R4532-R4535 (1996).

Cattaneo, F. and Vainshtein, S.I. "Suppression of turbulent transport by a weak magnetic field", Astrophys. J. Lett. 376, L21-L24 (1991).

Choudhuri, A.R. "Stochastic fluctuations of the solar dynamo", Astron. Astrophys., 253, 277-285 (1992).

Dittrich, P., Molchanov, S.A., Sokoloff, D.D. and Ruzmaikin, A.A. "Mean magnetic field in renovating random flow", Astron. Nachr. 305, 119-125 (1984).

Elperin, T., Kleeorin, N., Rogachevskii, I. and Sokoloff, D. "Passive scalar transport in a random flow with a finite renewal time: Mean-field equation", Phys. Rev. E 61, 2617-2625 (2000).

Field, G.B. and Blackman, E.G. "Dynamical quenching of the $\alpha^{2}$ dynamo", Astrophys. J. (in press) (2002) astro-ph/0111470.

Glatzmaier, G.A. and Roberts, P.H. “A three-dimensional self-consistent computer simulation of a geomagnetic field reversal", Nature 377, 203-209 (1995).

Hasler, K.-H., Kaisig, M. and Rüdiger, G. "Diffusion approximation probed with Parker instability simulations", Astrom. Astrophys. 295, 245-248 (1995).

Hawley, J.F., Gammie, C.F. and Balbus, S.A. "Local three-dimensional magnetohydrodynamic simulations of accretion discs", Astrophys. J. 440, 742-763 (1995).

Hawley, J.F., Gammie, C.F. and Balbus, S.A. "Local three dimensional simulations of an accretion disk hydromagnetic dynamo", Astrophys. J. 464, 690-703 (1996).

Hollerbach, R., Barenghi, C.F. and Jones, C.A. "Taylor constraint in a spherical alpha-omega-dynamo", Geophys. Astrophys. Fluid Dynam. 67, 3-25 (1992).

Hoyng, P., Schmitt, D. and Teuben, L.J.W. "The effect of random alpha-fluctuations and the global properties of the solar magnetic field", Astron. Astrophys. 289, 265-278 (1994).

Jones, C.A. and Wallace, S.G. "Periodic, chaotic and steady solutions in alpha-omega-dynamos", Geophys. Astrophys. Fluid Dynam. 67, 37-64 (1992).

Keinigs, R.K. "A new interpretation of the alpha effect”, Phys. Fluid 26, 2558-2560 (1983).

Kleeorin, N.I., Rogachevskii, I. and Ruzmaikin, A. "Magnitude of the dynamo-generated magnetic field in solar-type convective zones", Astron. Astrophys. 297, 159-167 (1995).

Krause, F. and Rädler, K.-H. Mean-Field Magnetohydrodynamics and Dynamo Theory. Akademie-Verlag, Berlin; also Pergamon Press, Oxford (1980). 
Miller, K.A. and Stone, J.M. "The formation and structure of a strongly magnetized corona above a weakly magnetized accretion disk", Astrophys. J. 534, 398-419 (2000).

Moffatt, H. K. "An approach to a dynamic theory of dynamo action in a rotating conducting fluid", J. Fluid Mech. 53, 385-399 (1972).

Moffatt, H.K. Magnetic Field Generation in Electrically Conducting Fluids. Cambridge University Press, Cambridge (1978).

Moffatt, H.K. "Transport effects associated with turbulence with particular attention to the influence of helicity”, Rep. Prog. Phys. 46, 621-664 (1983).

Molchanov, S.A., Ruzmaikin, A.A. and Sokoloff, D.D. "Kinematic dynamo in random flow", Sov. Phys. Usp. 28, 307-327 (1985).

Moss, D., Brandenburg, A., Tavakol, R.K. and Tuominen, I. "Stochastic effects in mean field dynamos", Astron. Astrophys. 265, 843-849 (1992).

Nicklaus, B. and Stix, M. "Corrections to first order smoothing in mean-field electrodynamics", Geophys. Astrophys. Fluid Dynam. 43, 149-166 (1988).

Ossendrijver, M., Stix, M. and Brandenburg, A. "Magnetoconvection and dynamo coefficients: dependence of the $\alpha$-effect on rotation and magnetic field", Astron. Astrophys. 376, 713-726 (2001).

Otmianowska-Mazur, K. "The turbulent EMF as a time series and the 'quality' of dynamo cycles", Geophys. Fluid Dynam. 86, 229-247 (1997).

Parker, E. N. "Hydromagnetic dynamo models", Astrophys. J. 122, 293-314 (1955).

Parker, E. N. Cosmical Magnetic Fields. Clarendon Press, Oxford (1979).

Rädler, K.-H., Apstein, E., Rheinhardt, M. and Schüler, M. "The Karlsruhe dynamo experiment. A mean field approach", Studia Geophys. et Geod. 42, 224-231 (1998).

Regös, E. "Parker's instability and viscosity in quiescent cataclysmic variable discs", Mon. Not. Roy. Astro. Soc. 286, 104-114 (1997).

Roberts, P.H. and Soward, A.M. "A unified approach to mean field electrodynamics", Astron. Nachr. 296, 49-64 (1975).

Rogachevskii, I. and Kleeorin, N. "Nonlinear turbulent magnetic diffusion and mean-field dynamo", Phys. Rev. E 64, 056307-1-14 (2001).

Rüdiger, G. "The influence of a uniform magnetic field of arbitrary strength on turbulence", Astron. Nachr. 295, 275-284 (1974).

Rüdiger, G. and Kitchatinov, L.L. "Alpha-effect and alpha-quenching”, Astron. Astrophys. 269, 581-588 (1993).

Rüdiger, G. and Pipin, V.V. "Viscosity-alpha and dynamo-alpha for magnetically driven compressible turbulence in Kepler disks", Astron. Astrophys. 362, 756-761 (2000).

Saar, S.H. and Brandenburg, A. "Time evolution of the magnetic activity cycle period. II. Results for an expanded stellar sample", Astrophys. J. 524, 295-310 (1999).

Steenbeck, M., Krause, F. and Rädler, K.-H. "Berechnung der mittleren Lorentz-Feldstärke $\overline{\mathbf{v} \times \mathbf{B}}$ für ein elektrisch leitendendes Medium in turbulenter, durch Coriolis-Kräfte beeinflußter Bewegung", $Z$. Naturforsch. 21a, 369-376 (1966). See also the translation in Roberts and Stix, The turbulent dynamo..., , Tech. Note 60, NCAR, Boulder, Colorado (1971).

Stefani, F., Gerbeth, G. and Rädler, K.-H. "Steady dynamos in finite domains: an integral equation approach", Astron. Nachr. 321, 65-73 (2000).

Stieglitz, R. and Müller, U. "Experimental demonstration of a homogeneous two-scale dynamo", Phys. Fluid 13, 561-564 (2001).

Tilgner, A. "Towards experimental fluid dynamos", Phys. Earth Planet. Int. 117, 171-177 (2000).

Tobias, S. "Relating stellar cycle periods to dynamo calculations", Mon. Not. Roy. Astro. Soc. 296, 653-661 (1998).

Tout, C.A. and Pringle, J.E. "Accretion disc viscosity: A simple model for a magnetic dynamo", Mon. Not. Roy. Astro. Soc. 259, 604-612 (1992).

Vainshtein, S.I. and Cattaneo, F. "Nonlinear restrictions on dynamo action", Astrophys. J. 393, 165-171 (1992).

Vishniac, E.T. and Brandenburg, A. "An incoherent $\alpha-\Omega$ dynamo in accretion disks", Astrophys. J. 475, 263-274 (1997).

Vishniac, E.T. and Cho, J. "Magnetic helicity conservation and astrophysical dynamos", Astrophys. J. 550, 752-760 (2001).

Zheligovsky, V.A., Podvigina, O.M. and Frisch, U. Dynamo effect in parity-invariant flow with large and moderate separation of scales", Geophys. Astrophys. Fluid Dynam. 95, 227-268 (2001).

Ziegler, U. and Rüdiger, G. "Angular momentum transport and dynamo-effect in stratified, weakly magnetic disks", Astron. Astrophys. 356, 1141-1148 (2000). 